\title{
Numerical Treatment of Initial-Boundary Value Problems with Mixed Boundary Conditions
}

\author{
Nawal Abdullah Alzaid, Huda Omar Bakodah \\ Department of Mathematics, King Abdulaziz University, Jeddah, KSA \\ Email:nalzaid@kau.edu.sa,hbakodah@kau.edu.sa
}

How to cite this paper: Alzaid, N.A. and Bakodah, H.O. (2018) Numerical Treatment of Initial-Boundary Value Problems with Mixed Boundary Conditions. American Journal of Computational Mathematics, 8, 153-174.

https://doi.org/10.4236/ajcm.2018.82012

Received: April 11, 2018

Accepted: June 25, 2018

Published: June 28, 2018

Copyright $\odot 2018$ by authors and Scientific Research Publishing Inc. This work is licensed under the Creative Commons Attribution International License (CC BY 4.0).

http://creativecommons.org/licenses/by/4.0/

\begin{abstract}
In this paper, we extend the reliable modification of the Adomian Decomposition Method coupled to the Lesnic's approach to solve boundary value problems and initial boundary value problems with mixed boundary conditions for linear and nonlinear partial differential equations. The method is applied to different forms of heat and wave equations as illustrative examples to exhibit the effectiveness of the method. The method provides the solution in a rapidly convergent series with components that can be computed iteratively. The numerical results for the illustrative examples obtained show remarkable agreement with the exact solutions. We also provide some graphical representations for clear-cut comparisons between the solutions using Maple software.

\section{Keywords}

Decomposition Method, Modified Adomian Decomposition Method, Linear and Nonlinear Partial Differential Equations, Mixed Boundary Conditions, Initial-Boundary Value Problem
\end{abstract}

\section{Introduction}

Mixed boundary value problems are characterized by a combination of Dirichlet and Neumann conditions along at least one boundary condition. They occur in a wide range of engineering and applied mathematics applications [1] [2] [3] [4]. These applications include the classic electrical potential and electric field conditions on a disk [5], stress and strain conditions around a punch pressing on an elastic surface [6] as well as some applications in porous media problems such as the infiltration and seepage among others [7] [8]. Historically, only very few of these problems could be solved using analytic methods. In view of this, many researchers obtained the solutions of initial and boundary value problems by 
using either initial or boundary condition(s). In recent years, there have been significant developments in the use of various semi-analytical methods for partial differential equations such as the homotopy perturbation method [9] and Adomian Decomposition Method (ADM) [10]. Duan and Rach [11] developed a new resolution method of Boundary Value Problems (BVPs) for nonlinear ordinary differential equations using the ADM. It is also well-known that the ADM provides approximate analytic solutions without using the Green function concept, which greatly facilitates analytic approximations and numerical computations. Several different resolution techniques for solving BVPs for nonlinear ordinary differential equations by using the ADM were considered by Adomian and Rach [12]-[18], Adomian [19], and Wazwaz [20]-[26]. Also, for a two-point BVP for second-order nonlinear differential equations, Adomian and Rach [17] [18] proposed the double decomposition method in order to avoid solving such nonlinear algebraic equations, and Jang [27] and Ebaid [28] introduced different modified inverse linear operators. Adomian [29] suggested a modified method for the hyperbolic, parabolic and elliptic partial differential equations with initial and boundary conditions by using two equations for $u$, one inverting the $L_{t}$ operator and the other inverting the $L_{x}$ operator, then, adding them and dividing by two. Further, with regards to the mixed value problems, Lesnic and Elliot [30] proposed the inverse operator defined by $L_{x}^{-1}=\int_{x_{0}}^{x} \mathrm{~d} x^{\prime} \int_{1}^{x} \mathrm{~d} x^{\prime \prime}$ to solve the linear homogeneous heat equation $u_{t}=u_{x x}, x_{0}<x<1, t>0$ subject to the mixed boundary conditions $u(x, t)=h_{1}(t), u_{x}(1, t)=h_{2}(t)$, where $\beta_{i}=(t), i=1,2$ are known functions. However, in this paper, we will present a modified recursion scheme based on the reliable modification of the ADM with new structure of the inverse operator applied to the (BVPs) with mixed boundary conditions using Lesnic's approach and Ebaid's method. The proposed operator allows the appearance of all the conditions in the solution thereby making the solution more realistic. The paper is arranged in the following manner: in Section 2, we analyze the ADM; Section 3 presents the modified ADM suggested by Wazwaz; in Section 4, Lesnic's approach is used to approximate solutions of some problems; the implementation of this new method to some test problems is presented in Section 5; finally, a brief conclusion is given in Section 6 .

\section{Analysis of the Adomian Decomposition Method with Mixed Conditions}

Nonlinear partial differential equations models in mathematics and physics play an important role in theoretical sciences. The understanding of these nonlinear partial differential equations is also crucial to many applied areas such as meteorology, oceanography, and aerospace industry. Nonlinear partial differential equations are the most fundamental models in studying nonlinear phenomena.

Consider the nonlinear partial differential equation given in an operator form

$$
L_{x} u(x, t)+L_{t} u(x, t)+R u(x, t)+N u(x, t)=g(x, t)
$$


where $L_{x}$ and $L_{t}$ are the linear operators to be inverted, which are usually the highest order differential operators in $x$, and $t$ respectively; $L_{x}, R$ is the linear remainder operator; $N u(x, t)$ is a nonlinear operator which is assumed to be analytic function, and $g(x, t)$ is the input function that is assumed to be continuous function. The solutions for $u(x, t)$ obtained from the operator equations $L_{x} u$ in $\mathrm{x}$-direction and $L_{t} u$ in $t$-direction are called partial solutions. We further give the following illustrations:

\subsection{Boundary Value Problems}

Consider the general form of the single second-order nonlinear inhomogeneous temporal-spatial partial differential equation:

$$
L_{x x} u(x, t)+L_{t t} u(x, t)+N u(x, t)=g(x, t), a \leq x \leq b, t>0,
$$

subject to the mixed boundary conditions

$$
u(a, t)=h_{1}(t), u_{x}(b, t)=h_{2}(t)
$$

where, $L_{x x} u(x, t)=\frac{\partial^{2}}{\partial x^{2}} u(x, t), L_{t t} u(x, t)=\frac{\partial^{2}}{\partial t^{2}} u(x, t)$. We consider the $x$ partial solution as

$$
L_{x x} u(x, t)=g(x, t)-L_{t t} u(x, t)-N u(x, t)
$$

Applying the two-fold indefinite integration inverse operator $L_{x x}^{-1}=\int \mathrm{d} x \int \mathrm{d} x^{\prime}$ to both sides of Equation (2.4), gives

$$
u(x, t)=\Phi_{x}+L_{x x}^{-1} g(x, t)-L_{x x}^{-1} L_{t t} u(x, t)-L_{x x}^{-1} N u(x, t),
$$

where $\Phi_{x}=c_{1}(t)+c_{2}(t) x$ and the constants of integrations $c_{1}(t)$ and $c_{2}(t)$, are determined from the boundary conditions. We now decompose the following $\Phi_{x}$, the linear and nonlinear terms $u$ and $N u$ based on ADM as follows:

$$
\begin{gathered}
\Phi_{x}=\sum_{n=0}^{\infty} \Phi_{x, n}=\sum_{n=0}^{\infty}\left(c_{1, n}(t)+c_{2, n}(t) x\right), \\
u(x, t)=\sum_{n=0}^{\infty} u_{n}(x, t), \\
N u(x, t)=\sum_{n=0}^{\infty} A_{n}\left(u_{0}, u_{1}, u_{2}, \cdots, u_{n}\right)
\end{gathered}
$$

where $A_{n}$ 's are the Adomian polynomials determined from the definitional formula

$$
A_{n}=\left.\frac{1}{n !} \frac{\mathrm{d}^{n}}{\mathrm{~d} \lambda^{n}} N\left(\sum_{k=0}^{\infty} u_{k} \lambda^{k}\right)\right|_{\lambda=0}, n \geq 0 .
$$

Substituting Equation (2.6) into Equation (2.5), yields the following recursion scheme

$$
\begin{gathered}
u_{0}=\Phi_{x, 0}+L_{x x}^{-1} g(x, t), \\
u_{n+1}=\Phi_{x, n+1}-L_{x x}^{-1} L_{t t} u_{n}-L_{x x}^{-1} A_{n}, n \geq 0 .
\end{gathered}
$$

The $n$-term approximation of the solution is $\varphi_{n}=\sum_{i=0}^{n-1} u_{i}(x, t)$ for $n>0$. 
Thus, $\varphi_{1}=u_{0}, \varphi_{2}=\varphi_{1}+u_{1}, \varphi_{3}=\varphi_{2}+u_{2}$, etc., and all $\varphi_{n}$ 's must satisfy the boundary conditions.

The first approximate $\varphi_{1}=u_{0}=c_{1,0}(t)+c_{2,0}(t) x+L_{x x}^{-1} g(x, t)$, where the values $c_{1}(t)$ and $c_{2}(t)$ can be evaluated by using the boundary conditions in Equation (2.2)

$$
\left.\varphi_{1}\right|_{x=a}=h_{1}(t),\left.\varphi_{1}^{\prime}\right|_{x=b}=h_{2}(t),
$$

which results in

$$
\begin{gathered}
c_{1,0}(t)+c_{2,0}(t) x+\left.L_{x x}^{-1} g(x, t)\right|_{x=a}=h_{1}(t), \\
c_{2,0}(t)+\left.\left(L_{x x}^{-1} g(x, t)\right)^{\prime}\right|_{x=b}=h_{2}(t) .
\end{gathered}
$$

Thus $\varphi_{1}$ is now determined. Since $u_{0}$ and $\varphi_{1}$ are now completely known, we form the next term $u_{1}=\Phi_{x, 1}-L_{x x}^{-1} L_{t t} u_{0}-L_{x x}^{-1} A_{0}$, then $\varphi_{2}=\varphi_{1}+u_{1}$, and we continue in the same manner to obtain $u_{2}, u_{3}, \cdots, u_{n}$ for some $n \geq 0$. Substituting all these values in $u(x, t)=\sum_{n=0}^{\infty} u_{n}(x, t)$, we get the solution of Equation (2.1).

\subsection{An Alternative Combination of the Initial and Boundary Conditions}

Adomian [29] suggested a modified method for the partial differential equations with initial and boundary conditions by using two equations for $u$, one inverting the $L_{t}$ operator and the other inverting the $L_{x}$ operator, then, adding them and dividing by two. To convey the basic idea for treatment of initial and boundary conditions by ADM for solving initial boundary value problems, we consider Equations (2.2)-(2.3) with the initial conditions

$$
u(x, 0)=p_{1}(x), u_{t}(x, 0)=p_{2}(x) .
$$

where $p_{i}(x), i=1,2$ are known functions.

Firstly, we consider the $t$ partial solution as

$$
L_{t t} u(x, t)=g(x, t)-L_{x x} u(x, t)-N u(x, t)
$$

Applying the inverse operator $L_{t t}^{-1}$ defined by $L_{t t}^{-1}=\int_{0}^{t} \int_{0}^{t} \mathrm{~d} t \mathrm{~d} t$ to both sides of Equation (2.10) and using the initial conditions, gives

$$
u(x, t)=u(x, 0)+t u_{t}(x, 0)+L_{t t}^{-1} g(x, t)-L_{t t}^{-1} L_{x x} u(x, t)-L_{t t}^{-1} N u(x, t)
$$

Secondly, we consider the $x$ partial solution as in Equation (2.5). Next, we averaged the partial solutions, i.e. add two partial solutions in Equation (2.5) and Equation (2.11) and divide by two, we obtain

$$
\begin{aligned}
u(x, t)= & \frac{1}{2}\left(u(x, 0)+t u_{t}(x, 0)+\Phi_{x}+L_{t t}^{-1} g(x, t)+L_{x x}^{-1} g(x, t)\right. \\
& \left.-L_{t t}^{-1} L_{x x} u(x, t)-L_{x x}^{-1} L_{t t} u(x, t)-L_{t t}^{-1} N u(x, t)-L_{x x}^{-1} N u(x, t)\right)
\end{aligned}
$$

Substituting Equation (2.6) into Equation (2.12), yields the following recursion scheme 


$$
\begin{gathered}
u_{0}=\frac{1}{2}\left(u(x, 0)+t u_{t}(x, 0)+\Phi_{x, 0}+L_{t t}^{-1} g(x, t)+L_{x x}^{-1} g(x, t)\right) \\
u_{n+1}=\frac{1}{2}\left(\Phi_{x, n}-L_{t t}^{-1} L_{x x} u_{n}(x, t)-L_{x x}^{-1} L_{t t} u_{n}(x, t)-L_{t t}^{-1} A_{n}-L_{x x}^{-1} A_{n}\right), n \geq 0 .
\end{gathered}
$$

To illustrate this method for coupled linear and nonlinear partial differential equations, we take two examples in the following section.

\subsection{Numerical Experiments}

\section{Example 1.}

Consider the linear homogeneous heat equation

$$
u_{t}-u_{x x}=0,0 \leq x \leq 1, t>0,
$$

with specified conditions $u(0, t)=0, u_{x}(1, t)=\cos (1) \mathrm{e}^{-t}$.

Rewriting the heat equation in the operator form as $L_{t} u-L_{x x} u=0$.

Applying the inverse operator $L_{x x}^{-1}$ defined by $L_{x x}^{-1}=\int \mathrm{d} x \int \mathrm{d} x^{\prime}$, gives

$$
u(x, t)=c_{1}(t)+c_{2}(t) x+L_{x x}^{-1} L_{t} u(x, t) .
$$

This introduces the recursive relations

$$
\begin{gathered}
u_{0}=c_{1}(t)+c_{2}(t) x, \\
u_{n+1}=c_{1, n+1}(t)+c_{2, n+1}(t) x+L_{x x}^{-1} L_{t} u_{n}, n \geq 0 .
\end{gathered}
$$

The first approximant is $\varphi_{1}=u_{0}=c_{1,0}(t)+c_{2,0}(t) x$. Applying the $x$ conditions to $\varphi_{1}$, it is clear that $c_{1,0}(t)=0$ and $c_{2,0}(t)=\cos (1) \mathrm{e}^{-t}$. Thus, if the one-term approximant $\varphi_{1}$ were sufficient, the solution would be $\varphi_{1}=u_{0}=\cos (1) \mathrm{e}^{-t} x$, The next term is

$$
\begin{aligned}
u_{1} & =c_{1,1}(t)+c_{2,1}(t) x+L_{x x}^{-1} L_{t} u_{0} \\
& =c_{1,1}(t)+c_{2,1}(t) x+L_{x x}^{-1} L_{t}\left(\cos (1) \mathrm{e}^{-t} x\right)
\end{aligned}
$$

Then $\varphi_{2}=u_{0}+u_{1}$ is given by

$$
\varphi_{2}=\cos (1) \mathrm{e}^{-t} x+c_{1,1}(t)+c_{2,1}(t) x-\frac{1}{6} \cos (1) \mathrm{e}^{-t} x^{3}
$$

Applying the condition at $x=0$, we have $c_{1,1}(t)=0$. From the condition on $x$ at 1 , we get

$$
c_{2,1}(t)=\frac{1}{2} \cos (1) \mathrm{e}^{-t}, \text { thus } u_{1}=\frac{1}{2} \cos (1) \mathrm{e}^{-t} x-\frac{1}{6} \cos (1) \mathrm{e}^{-t} x^{3}
$$

Continuing in a similar way $u_{2}, u_{3}, \cdots, u_{n}$ are obtained for some $n$, then we get the approximate solution $u_{a p}=\sum_{n=0}^{\infty} u_{n}(x, t)$ which converged to the exact solution $u_{e x}=\cos (x) \mathrm{e}^{-t}$.

Table 1 shows the comparison between the absolute error of the exact and approximate solutions for various values of $t$. Figure 1 and Figure 2 give the plots for the exact and approximate solutions by using ADM for $t=0.5, t=1.0$ and $0 \leq x \leq 1$, respectively. 
Table 1. Absolute errors using ADM at $t=0.5, t=1.0$ and $0 \leq x \leq 1$.

\begin{tabular}{ccccccc}
\hline & \multicolumn{3}{c}{$T=0.5$} & & & $T=1.0$ \\
\cline { 2 - 6 } & $\varphi_{3}$ & $\varphi_{5}$ & $\varphi_{10}$ & $\varphi_{3}$ & $\varphi_{5}$ & $\varphi_{10}$ \\
\hline 0.0 & $0.00000000 \mathrm{e}+00$ & $0.00000000 \mathrm{e}+00$ & $0.00000000 \mathrm{e}+00$ & $0.00000000 \mathrm{e}+00$ & $0.00000000 \mathrm{e}+00$ & $0.00000000 \mathrm{e}+00$ \\
0.1 & $4.65015125 \mathrm{e}-03$ & $7.64016845 \mathrm{e}-04$ & $8.35421477 \mathrm{e}-06$ & $2.82045931 \mathrm{e}-03$ & $4.63399641 \mathrm{e}-04$ & $5.06708740 \mathrm{e}-06$ \\
0.2 & $9.18603225 \mathrm{e}-03$ & $1.50922156 \mathrm{e}-03$ & $1.65027211 \mathrm{e}-05$ & $5.57161020 \mathrm{e}-03$ & $9.15389147 \mathrm{e}-04$ & $1.00094063 \mathrm{e}-05$ \\
0.3 & $1.34961408 \mathrm{e}-02$ & $2.21726512 \mathrm{e}-03$ & $2.42448756 \mathrm{e}-05$ & $8.18582316 \mathrm{e}-03$ & $1.34483927 \mathrm{e}-03$ & $1.47052604 \mathrm{e}-05$ \\
0.4 & $1.74744502 \mathrm{e}-02$ & $2.87071334 \mathrm{e}-03$ & $3.13900408 \mathrm{e}-05$ & $1.05987898 \mathrm{e}-02$ & $1.74117565 \mathrm{e}-03$ & $1.90390221 \mathrm{e}-05$ \\
0.5 & $2.10229970 \mathrm{e}-02$ & $3.45347612 \mathrm{e}-03$ & $3.77622790 \mathrm{e}-05$ & $1.27510922 \mathrm{e}-02$ & $2.09463915 \mathrm{e}-03$ & $2.29039800 \mathrm{e}-05$ \\
0.6 & $2.40542893 \mathrm{e}-02$ & $3.95120365 \mathrm{e}-03$ & $4.32046846 \mathrm{e}-05$ & $1.45896640 \mathrm{e}-02$ & $2.39652615 \mathrm{e}-03$ & $2.62049659 \mathrm{e}-05$ \\
0.7 & $2.64934801 \mathrm{e}-02$ & $4.35163977 \mathrm{e}-03$ & $4.75832475 \mathrm{e}-05$ & $1.60691079 \mathrm{e}-02$ & $2.63940294 \mathrm{e}-03$ & $2.88606985 \mathrm{e}-05$ \\
0.8 & $2.82802497 \mathrm{e}-02$ & $4.64492392 \mathrm{e}-03$ & $5.07901529 \mathrm{e}-05$ & $1.71528385 \mathrm{e}-02$ & $2.81728877 \mathrm{e}-03$ & $3.08057849 \mathrm{e}-05$ \\
0.9 & $2.93703477 \mathrm{e}-02$ & $4.82383397 \mathrm{e}-03$ & $5.27464362 \mathrm{e}-05$ & $1.78140164 \mathrm{e}-02$ & $2.92580320 \mathrm{e}-03$ & $3.19923307 \mathrm{e}-05$ \\
1.0 & $2.97367443 \mathrm{e}-02$ & $4.88396424 \mathrm{e}-03$ & $5.34039271 \mathrm{e}-05$ & $1.80362471 \mathrm{e}-02$ & $2.96227405 \mathrm{e}-03$ & $3.23911192 \mathrm{e}-05$ \\
\hline
\end{tabular}
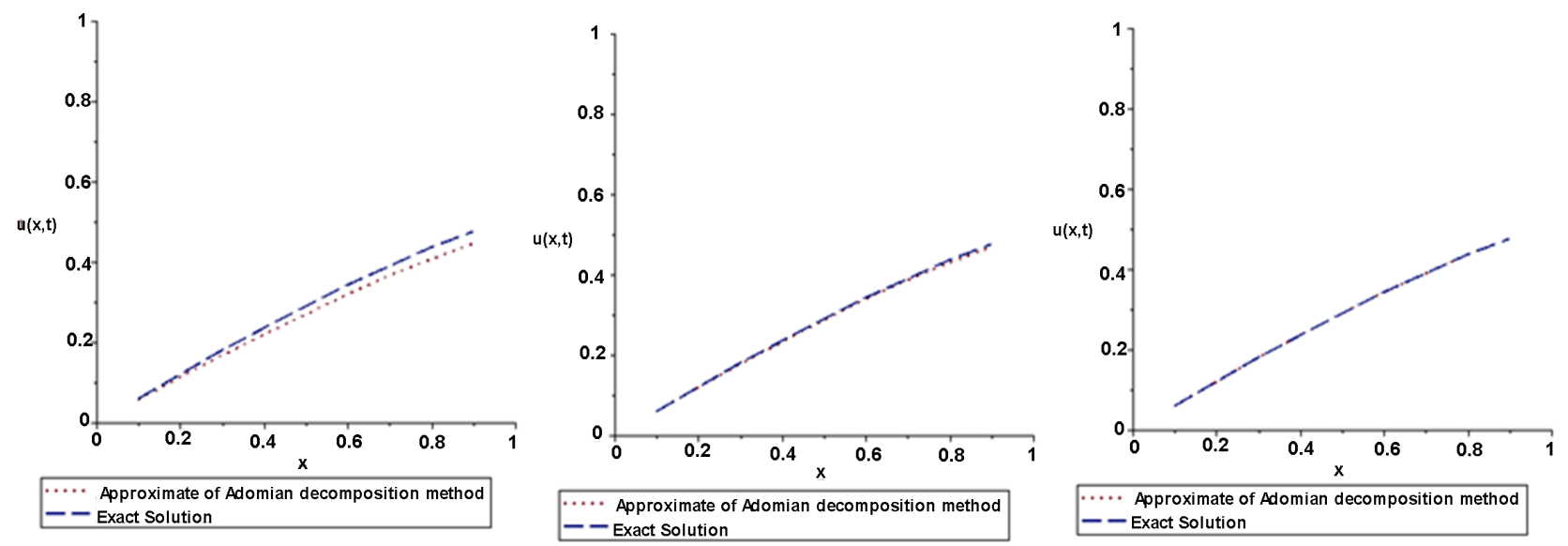

Figure 1. The exact solution and the approximate solution using $\mathrm{ADM}$ for $\varphi_{3}, \varphi_{5}, \varphi_{10}$ at $t=0.5$ and $0 \leq x \leq 1$.

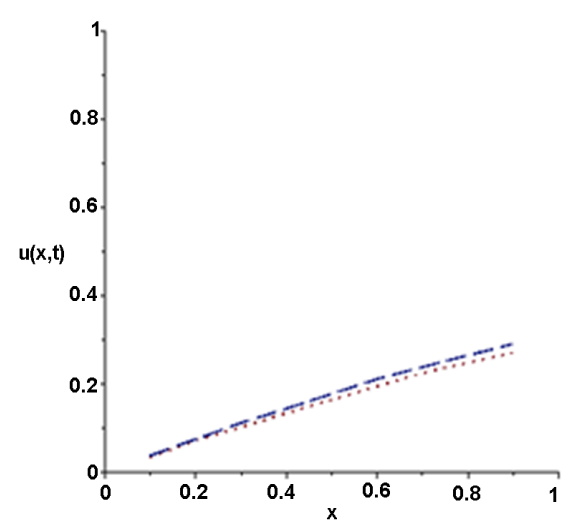

$\cdots$ Approximate of Adomian decomposition method
-- Exact Solution

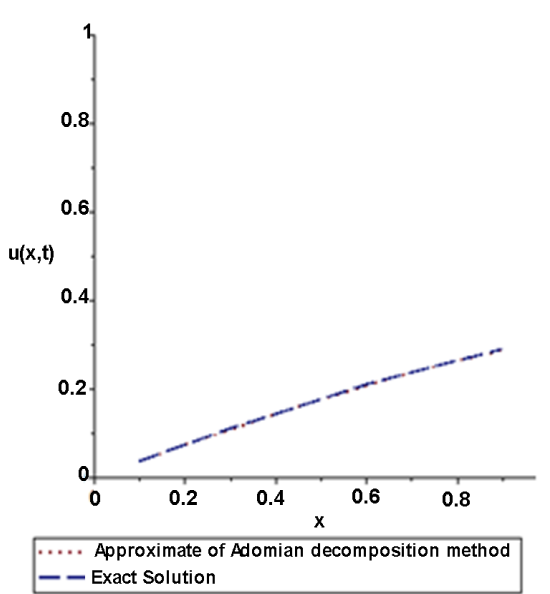

Figure 2. The exact solution and the approximate solution using $\mathrm{ADM}$ for $\varphi_{3}, \varphi_{5}, \varphi_{10}$ at $t=1.0$ and $0 \leq x \leq 1$. 


\section{Example 2}

Consider the nonlinear inhomogeneous wave equation

$$
u_{t t}-u_{x x}-u+u^{2}=x t+x^{2} t^{2}, t>0,
$$

with specified initial conditions $u(x, 0)=1, u_{t}(x, 0)=x$, and the boundary conditions $u(0, t)=1, u_{x}(0, t)=t$. Rewriting the wave equation in the operator form as

$$
L_{t t} u-L_{x x} u-u+u^{2}=x t+x^{2} t^{2} .
$$

To solve initial-boundary value problem, firstly, we consider the $t$ partial solution as $L_{t t} u=L_{x x} u+u-u^{2}+x t+x^{2} t^{2}$.

Applying the inverse operator $L_{t t}^{-1}$ defined by $L_{t t}^{-1}=\int_{0}^{t} \int_{0}^{t} \mathrm{~d} t \mathrm{~d} t$, gives

$$
u(x, t)=u(x, 0)+t u_{t}(x, 0)+L_{t t}^{-1}\left(x t+x^{2} t^{2}\right)+L_{t t}^{-1} L_{x x} u(x, t)+L_{t t}^{-1} u-L_{t t}^{-1} u^{2} .
$$

This introduces the recursive relations

$$
\begin{gathered}
u_{0}=u(x, 0)+t u_{t}(x, 0)+L_{t t}^{-1}\left(x t+x^{2} t^{2}\right) \\
u_{n+1}=L_{t t}^{-1} L_{x x} u_{n}+L_{t t}^{-1} u_{n}-L_{t t}^{-1} A_{n}, n \geq 0 .
\end{gathered}
$$

where the nonlinear term $u^{2}$ can be expressed by an infinite series of the Adomian polynomials $A_{n}$ given by:

$$
\begin{gathered}
A_{0}=\left(u_{0}\right)^{2}, \\
A_{1}=2 u_{0} u_{1}, \\
A_{2}=\left(u_{1}\right)^{2}+2 u_{0} u_{2}, \\
A_{3}=2 u_{1} u_{2}+2 u_{0} u_{3}, \\
A_{4}=\left(u_{2}\right)^{2}+2 u_{1} u_{3}+2 u_{0} u_{4},
\end{gathered}
$$

So that first two terms are

$$
\begin{gathered}
u_{0}=1+x t+\frac{1}{6} x t^{3}+\frac{1}{12} x^{2} t^{4}, \\
u_{1}=\frac{1}{180} t^{6}-\frac{1}{12960} x^{4} t^{10}-\frac{1}{2529} x^{3} t^{9}-\frac{1}{2016} x^{2} t^{8}-\frac{1}{252} x^{3} t^{7} \\
-\frac{1}{72} x^{2} t^{6}-\frac{1}{120} x t^{5}-\frac{1}{12} x^{2} t^{4}-\frac{1}{6} x t^{3},
\end{gathered}
$$

Secondly, we consider the $x$ partial solution $L_{x x} u=L_{t t} u-u+u^{2}-x t-x^{2} t^{2}$.

Applying the inverse operator $L_{x x}^{-1}$ defined by $L_{x x}^{-1}=\int \mathrm{d} x \int \mathrm{d} x^{\prime}$, gives

$$
u(x, t)=\Phi_{x}+L_{x x}^{-1}\left(-x t-x^{2} t^{2}\right)+L_{x x}^{-1} L_{t t} u(x, t)-L_{x x}^{-1} u(x, t)+L_{x x}^{-1} u^{2}(x, t),
$$

This gives the recursive relations

$$
\begin{gathered}
u_{0}=\Phi_{x, 0}+L_{x x}^{-1}\left(-x t-x^{2} t^{2}\right), \\
u_{n+1}=\Phi_{x, n}+L_{x x}^{-1} L_{t t} u_{n}-L_{x x}^{-1} u_{n}+L_{x x}^{-1} A_{n}, n \geq 0 .
\end{gathered}
$$

So that 


$$
\begin{aligned}
u_{0} & =c_{1,0}(t)+c_{2,0}(t) x+L_{x x}^{-1}\left(-x t-x^{2} t^{2}\right) \\
& =c_{1,0}(t)+c_{2,0}(t) x-\frac{1}{12} x^{4} t^{2}-\frac{1}{6} x^{3} t,
\end{aligned}
$$

The first approximant is $\varphi_{1}=u_{0}$. Applying the $x$ conditions to $\varphi_{1}$ it is clear that $c_{1,0}(t)=1$ and $c_{2,0}(t)=t$. Thus, if the one-term approximant $\varphi_{1}$ were sufficient, the solution would be $\varphi_{1}=u_{0}=1+x t-\frac{1}{12} x^{4} t^{2}-\frac{1}{6} x^{3} t$. The next term is

$$
u_{1}=c_{1,1}(t)+c_{2,1}(t) x+L_{x x}^{-1} L_{t t} u_{0}-L_{x x}^{-1} u_{0}+L_{x x}^{-1} A_{0} .
$$

Then $\varphi_{2}=u_{0}+u_{1}$ is given by:

$$
\begin{aligned}
\varphi_{2}= & 1+x t+c_{1,1}(t)+c_{2,1}(t) x-\frac{1}{180} x^{6}+\frac{1}{12960} t^{4} x^{10}+\frac{1}{2592} t^{3} x^{9} \\
& +\frac{1}{2016} t^{2} x^{8}-\frac{1}{252} t^{3} x^{7}-\frac{1}{72} t^{2} x^{6}-\frac{1}{120} t x^{5},
\end{aligned}
$$

Applying the condition at $x=0$, we have $c_{1,1}(t)=0$. From the condition on $x$ at 0 , we get $c_{2,1}(t)=0$, thus

$$
\begin{aligned}
u_{1}= & -\frac{1}{180} x^{6}+\frac{1}{12960} t^{4} x^{10}+\frac{1}{2592} t^{3} x^{9}+\frac{1}{2016} t^{2} x^{8} \\
& -\frac{1}{252} t^{3} x^{7}-\frac{1}{72} t^{2} x^{6}-\frac{1}{120} t x^{5}+\frac{1}{12} t^{2} x^{4}+\frac{1}{6} t x^{3},
\end{aligned}
$$

Next, we average the partial solutions, i.e. add two partial solutions and divide by two, so we obtain

$$
\begin{gathered}
u_{0}=\frac{1}{2}\left(2+2 x t+\frac{1}{12} x^{2} t^{4}+\frac{1}{6} x t^{3}-\frac{1}{12} x^{4} t^{2}-\frac{1}{6} x^{3} t\right) \\
u_{1}=\frac{1}{2}\left(\frac{1}{180} t^{6}-\frac{1}{12960} x^{4} t^{10}-\frac{1}{2592} x^{3} t^{9}-\frac{1}{2016} x^{2} t^{8}-\frac{1}{252} x^{3} t^{7}-\frac{1}{72} x^{2} t^{6}\right. \\
-\frac{1}{120} x t^{5}-\frac{1}{12} x^{2} t^{4}-\frac{1}{6} x t^{3}-\frac{1}{180} x^{6}+\frac{1}{12960} t^{4} x^{10}+\frac{1}{2592} t^{3} x^{9} \\
\left.+\frac{1}{2016} t^{2} x^{8}-\frac{1}{252} t^{3} x^{7}-\frac{1}{72} t^{2} x^{6}-\frac{1}{120} t x^{5}+\frac{1}{12} t^{2} x^{4}+\frac{1}{6} t x^{3}\right)
\end{gathered}
$$

Continuing in a similar way $u_{2}, u_{3}, \cdots, u_{n}$ are obtained for some $\mathrm{n}$, then we get the approximate solution $u_{a p}=\sum_{n=0}^{\infty} u_{n}(x, t)$ which is converge to the exact solution $u_{e x}=1+x t$.

Table 2 shows the comparison between the absolute error of exact and approximate solutions for various values of $t$. Figure 3 and Figure 4 give the plots for the exact solution and the approximate solution by application of (ADM) for $t=0.5, t=1.0$

\section{Modified Adomian Decomposition Method with Mixed Conditions}

Wazwaz in [31], suggested a modification to the recurrence relations of the Standard Adomian Decomposition Method (ADM). He assumed that the first 
Table 2. Absolute errors using ADM at $t=0.5, t=1.0$ and $0 \leq x \leq 1$.

\begin{tabular}{|c|c|c|c|c|c|c|}
\hline \multirow{2}{*}{$x$} & \multicolumn{3}{|c|}{$t=0.5$} & \multicolumn{3}{|c|}{$t=1.0$} \\
\hline & $\varphi_{3}$ & $\varphi_{5}$ & $\varphi_{10}$ & $\varphi_{3}$ & $\varphi_{5}$ & $\varphi_{10}$ \\
\hline 0.0 & $1.16795669 \mathrm{e}-06$ & $9.91381644 \mathrm{e}-11$ & $7.38008938 \mathrm{e}-21$ & $3.03130511 \mathrm{e}-04$ & $1.94227703 \mathrm{e}-07$ & $1.98867397 \mathrm{e}-13$ \\
\hline 0.1 & $1.11741220 \mathrm{e}-06$ & $1.34624036 \mathrm{e}-10$ & $1.32310675 \mathrm{e}-20$ & $3.13576647 \mathrm{e}-04$ & $5.24225154 \mathrm{e}-07$ & $1.80979784 \mathrm{e}-14$ \\
\hline 0.2 & $1.02259378 \mathrm{e}-06$ & $1.68442683 \mathrm{e}-10$ & $1.87267309 \mathrm{e}-20$ & $3.10560067 \mathrm{e}-04$ & $8.92768322 \mathrm{e}-07$ & $3.35618411 \mathrm{e}-13$ \\
\hline 0.3 & $9.15093801 \mathrm{e}-07$ & $1.98929868 \mathrm{e}-10$ & $2.27716130 \mathrm{e}-20$ & $2.91108055 \mathrm{e}-04$ & $1.27778455 \mathrm{e}-06$ & $7.19015636 \mathrm{e}-13$ \\
\hline 0.4 & $1.07394957 \mathrm{e}-06$ & $2.47291825 \mathrm{e}-10$ & $2.45567197 \mathrm{e}-20$ & $2.52542863 \mathrm{e}-04$ & $1.64754249 \mathrm{e}-06$ & $1.09372711 \mathrm{e}-12$ \\
\hline 0.5 & $2.78526691 \mathrm{e}-06$ & $6.80058898 \mathrm{e}-10$ & $1.54926759 \mathrm{e}-19$ & $1.94233658 \mathrm{e}-04$ & $1.95990772 \mathrm{e}-06$ & $1.34001536 \mathrm{e}-12$ \\
\hline 0.6 & $1.02972732 \mathrm{e}-05$ & $4.84484741 \mathrm{e}-09$ & $1.31137692 \mathrm{e}-17$ & $1.22320508 \mathrm{e}-04$ & $2.16910519 \mathrm{e}-06$ & $1.29712536 \mathrm{e}-12$ \\
\hline 0.7 & $3.49347643 \mathrm{e}-05$ & $3.38038332 \mathrm{e}-08$ & $6.42509911 \mathrm{e}-16$ & $6.00678450 \mathrm{e}-05$ & $2.27490316 \mathrm{e}-06$ & $7.88380042 \mathrm{e}-13$ \\
\hline 0.8 & $1.02662323 \mathrm{e}-04$ & $1.89000465 \mathrm{e}-07$ & $1.89050311 \mathrm{e}-14$ & $6.75505137 \mathrm{e}-05$ & $2.54957010 \mathrm{e}-06$ & $2.03620129 \mathrm{e}-13$ \\
\hline 0.9 & $2.66686450 \mathrm{e}-04$ & $8.69375166 \mathrm{e}-07$ & $3.75110306 \mathrm{e}-13$ & $2.75330767 \mathrm{e}-04$ & $4.36439340 \mathrm{e}-06$ & $1.01270536 \mathrm{e}-12$ \\
\hline 1.0 & $6.26917478 \mathrm{e}-04$ & $3.41245963 \mathrm{e}-06$ & $5.43693717 \mathrm{e}-12$ & $9.37415208 \mathrm{e}-04$ & $1.27237836 \mathrm{e}-05$ & $4.38984895 \mathrm{e}-11$ \\
\hline
\end{tabular}

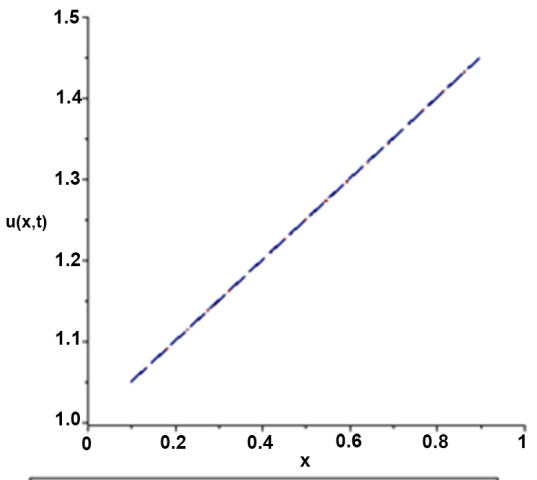

… Approximate of Adomian decomposition method - Exact Solution
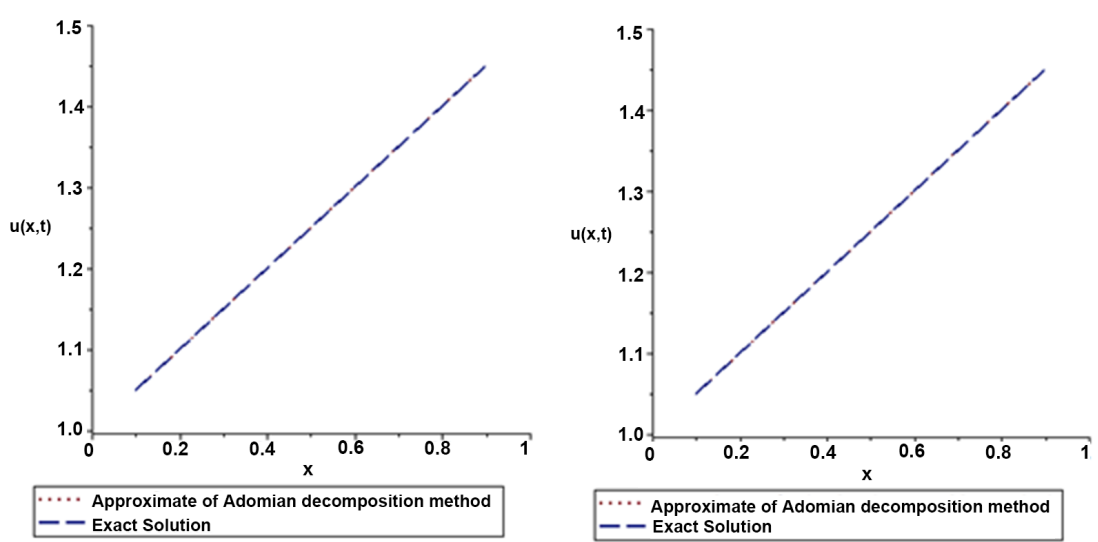

Figure 3. The exact solution and the approximate solution using ADM for $\varphi_{3}, \varphi_{5}, \varphi_{10}$ at $t=0.5$.

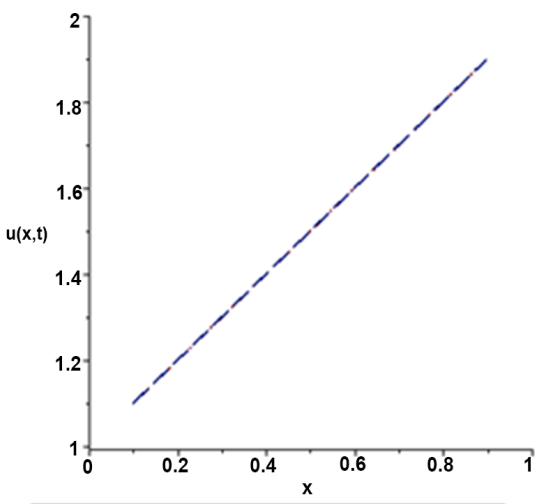

..... Approximate of Adomian decomposition method - Exact Solution

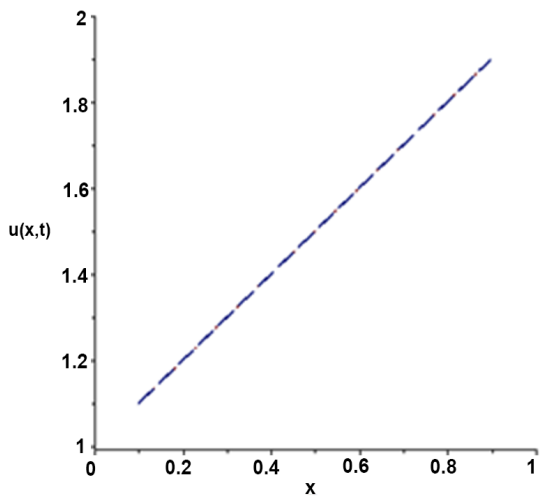

$\cdots$... Approximate of Adomian decomposition method - Exact Solution

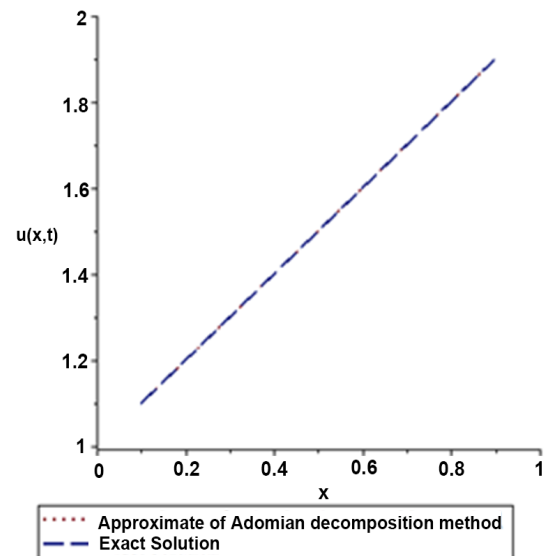

- Exact Solution

Figure 4. The exact solution and the approximate solution using ADM for $\varphi_{3}, \varphi_{5}, \varphi_{10}$ at $t=1.0$. 
term $u_{0}$ can be set as function $f$ and divided it into two parts, namely $f_{1}$ and $f_{2}$. Under this assumption, we set $f=f_{1}+f_{2}$. Also, based on this, we propose a slight variation only on the components $u_{0}$ and $u_{1}$, the variation is that only the part $f_{1}$ is assigned to the zeroth component $u_{0}$, whereas the remaining part $f_{2}$ is combined with the other terms to define $u_{1}$. This reduction in the number of terms of $u_{0}$ will result in reduction of the computational work and will accelerate the convergence. Further, this slight variation in the definition of the components $u_{0}$ and $u_{1}$ may provide the solution by using two iterations only. Furthermore, the calculations below will show that sometimes there is no need to evaluate the so-called Adomian polynomials required for the nonlinear differential equations. An important observation that can be made here is that the success of this method depends mainly on the proper choice of the parts $f_{1}$ and $f_{2}$. We have been unable to establish any criterion to judge what forms of $f_{1}$ and $f_{2}$ can be assumed to yield the acceleration demanded. It appears that trials are the only criteria that can be applied so far.

\subsection{Boundary Value Problems}

Based on the recurrence relation in Equation (2.8)

$$
\begin{gathered}
u_{0}=\Phi_{x, 0}+L_{x x}^{-1} g(x, t), \\
u_{n+1}=\Phi_{x, n+1}-L_{x x}^{-1} L_{t t} u_{n}-L_{x x}^{-1} A_{n}, n \geq 0 .
\end{gathered}
$$

We can set $u_{0}=\Phi_{x, 0}+L_{x x}^{-1} g(x, t)=f$, then we divide it into two parts, so that we formulate the modified recursive algorithm as follows:

$$
\begin{gathered}
u_{0}=f_{1}, \\
u_{1}=f_{2}+\Phi_{x, 1}-L_{x x}^{-1} L_{t t} u_{0}-L_{x x}^{-1} A_{0}, \\
u_{n+1}=\Phi_{x, n+1}-L_{x x}^{-1} L_{t t} u_{n}-L_{x x}^{-1} A_{n}, n \geq 1 .
\end{gathered}
$$

Comparing the recursive scheme in Equation (2.8) of the ADM with the scheme in Equation (3.1) of the modified technique leads to the conclusion that in Equation (2.8) the zeroth component was defined by the function $f$, whereas in Equation (3.1), the zeroth component $u_{0}$ was defined by only a part $f_{1}$ of $f$, the remaining part $f_{2}$ of $f$ is added to the definition of the component $u_{1}$ in (3.1).

\subsection{An alternative Combination of the Initial and Boundary Conditions}

Based on the recurrence relation in Equation (2.13), we can set

$$
u_{0}=\frac{1}{2}\left(u(x, 0)+t u_{t}(x, 0)+\Phi_{x, 0}+L_{t t}^{-1} g(x, t)+L_{x x}^{-1} g(x, t)\right)=f
$$

Then we divide it into two parts, so that we formulate the modified recursive algorithm as follows:

$$
\begin{gathered}
u_{0}=f_{1}, \\
u_{1}=f_{2}+\frac{1}{2}\left(\Phi_{x, 1}-L_{t t}^{-1} L_{x x} u_{0}-L_{x x}^{-1} L_{t t} u_{0}-L_{t t}^{-1} A_{0}-L_{x x}^{-1} A_{0}\right),
\end{gathered}
$$




$$
u_{n+1}=\frac{1}{2}\left(\Phi_{x, n+1}-L_{t t}^{-1} L_{x x} u_{n}-L_{x x}^{-1} L_{t t} u_{n}-L_{t t}^{-1} A_{n}-L_{x x}^{-1} A_{n}\right), n \geq 1 .
$$

Comparing the recursive scheme in Equation. (2.13) of the ADM with the recursive scheme in Equation (3.2) of the modified technique leads to the conclusion that in (2.13) the zeroth component was defined by the function $f$, whereas in (3.2), the zeroth component $u_{0}$ is defined only by a part $f_{1}$ of $f$, the remaining part $f_{2}$ of $f$ is added to the definition of the component $u_{1}$ in (2.13).

In order to demonstrate the efficiency and applicability of the proposed method, we study example of nonlinear partial differential equations here.

\subsection{Numerical Experiments}

\section{Example 3}

Consider the nonlinear inhomogeneous heat equation

$$
u_{t}=u_{x x}+u^{2}-x^{2} t^{2}+x, t>0 \text {, }
$$

with specified conditions $u(x, 0)=0, u(0, t)=0, u_{x}(0, t)=t$.

Rewriting the heat equation in the operator form as

$$
L_{t} u=L_{x x} u+u^{2}-x^{2} t^{2}+x .
$$

Applying the inverse operator $L_{x x}^{-1}$ defined by $L_{x x}^{-1}=\int \mathrm{d} x \int \mathrm{d} x^{\prime}$, we get

$$
u(x, t)=\Phi_{x}+L_{x x}^{-1}\left(x^{2} t^{2}-x\right)+L_{x x}^{-1} L_{t} u(x, t)-L_{x x}^{-1} u^{2}(x, t),
$$

This gives the recursive relations

$$
\begin{gathered}
u_{0}=\Phi_{x, 0}+L_{x x}^{-1}\left(x^{2} t^{2}-x\right), \\
u_{n+1}=\Phi_{x, n+1}+L_{x x}^{-1} L_{t} u_{n}-L_{x x}^{-1} A_{n}, n \geq 0 .
\end{gathered}
$$

So that

$$
\begin{aligned}
u_{0} & =c_{1,0}(t)+c_{2,0}(t) x+L_{x x}^{-1}\left(x^{2} t^{2}-x\right) \\
& =c_{1,0}(t)+c_{2,0}(t) x+\frac{1}{12} x^{4} t^{2}-\frac{1}{6} x^{3},
\end{aligned}
$$

The first approximant is $\varphi_{1}=u_{0}$ Applying the $x$ conditions to $\varphi_{1}$ it is clear that $c_{1,0}(t)=0$ and $c_{2,0}(t)=t$. Thus, if the one-term approximant $\varphi_{1}$ were sufficient, the "solution" will be $\varphi_{1}=u_{0}=x t+\frac{1}{12} x^{4} t^{2}-\frac{1}{6} x^{3}$. We set $u_{0}=f$, then we divide it into two parts, so that

$$
\begin{gathered}
u_{0}=f_{1}=x t, \\
u_{1}=f_{2}+c_{1,1}(t)+c_{2,1}(t) x+L_{x x}^{-1} L_{t} u_{0}-L_{x x}^{-1} A_{0} \\
=\left(\frac{1}{12} x^{4} t^{2}-\frac{1}{6} x^{3}\right)+c_{1,1}(t)+c_{2,1}(t) x+L_{x x}^{-1} L_{t} u_{0}-L_{x x}^{-1} A_{0} .
\end{gathered}
$$

Then $\varphi_{2}=u_{0}+u_{1}$ is given by:

$$
\varphi_{2}=x t+\frac{1}{12} x^{4} t^{2}-\frac{1}{6} x^{3}+c_{1,1}(t)+c_{2,1}(t) x+L_{x x}^{-1} L_{t} u_{0}-L_{x x}^{-1} A_{0}
$$

Applying the condition at $x=0$, we have $c_{1,1}(t)=0$. From the condition on 
$x$ at 0 , we get $c_{2,1}(t)=0$, thus $u_{1}=0$, and $u_{n+1}=0, n \geq 1$. Thus, the approximate solution is $u=u_{0}=x t$, which is the exact solution.

\section{Improvement of the Inverse Operator with Mixed Boundary Conditions}

In 1999, Lesnic and Elliott [30] employed ADM for solving some inverse boundary value problems in heat conduction, also they applied the modification method to deal with noisy input data and obtain a stable approximate solution. In [32] Lesnic investigated the application of the decomposition method involving computational algebra for solving more complicated problems with Dirichlet, Neumann or mixed boundary conditions. In [30] the authors defined the operator $L_{t}^{-1}$ and as definite integrals given by

$$
L_{t}^{-1}=\int_{0}^{t} \mathrm{~d} t^{\prime}, L_{x}^{-1}=\int_{x_{0}}^{x} \mathrm{~d} x^{\prime} \int_{1}^{x^{\prime}} \mathrm{d} x^{\prime \prime}
$$

to solve the linear homogeneous heat equation $u_{t}=u_{x x}, x_{0}<x<1, t>0$, subject to the mixed boundary conditions $u\left(x_{0}, t\right)=h_{1}(t), u_{x}(1, t)=h_{2}(t)$.

We will make an extension to the inverse operator (4.1) given in [30] to all cases of problems, so that we consider in this section, two types of problems: boundary value problems and initial-boundary value problems.

\subsection{Boundary Value Problems}

Consider Equations. (2.2)-(2.3), and applying the inverse operator $L_{x x}^{-1}$ defined by

$$
\begin{aligned}
& L_{x x}^{-1}=\int_{a}^{x} \mathrm{~d} x^{\prime} \int_{b}^{x^{\prime}} \mathrm{d} x^{\prime \prime} \quad \text { to both sides of Equation (2.4), gives } \\
& u(x, t)=u(a, t)+(x-a) \frac{\partial u(b, t)}{\partial x}+L_{x x}^{-1} g(x, t)-L_{x x}^{-1} L_{t t} u(x, t)-L_{x x}^{-1} N u(x, t)
\end{aligned}
$$

i.e. the boundary conditions can be used directly to solve the boundary value problem in $x$-direction.

Substituting $u(x, t)=\sum_{n=0}^{\infty} u_{n}(x, t)$, and $N u(x, t)=\sum_{n=0}^{\infty} A_{n}\left(u_{0}, u_{1}, u_{2}, \cdots, u_{n}\right)$ into Equation (4.2), gives

$$
\begin{aligned}
\sum_{n=0}^{\infty} u_{n}(x, t)= & u(a, t)+(x-a) \frac{\partial u(b, t)}{\partial x}+L_{x x}^{-1} g(x, t) \\
& -L_{x x}^{-1} L_{t t} \sum_{n=0}^{\infty} u_{n}(x, t)-L_{x x}^{-1} \sum_{n=0}^{\infty} A_{n}(x, t)
\end{aligned}
$$

This yields the recursive relations

$$
\begin{gathered}
u_{0}=u(a, t)+(x-a) \frac{\partial u(b, t)}{\partial x}+L_{x x}^{-1} g(x, t), \\
u_{n+1}=-L_{x x}^{-1} L_{t t} u_{n}-L_{x x}^{-1} A_{n}, n \geq 0 .
\end{gathered}
$$

\subsection{An Alternative Combination of the Initial and Boundary Conditions}

Here, we take the average of the $t$ partial solution as in Equation (2.11), and the $x$ 
partial solution as in Equation (4.2) to get

$$
\begin{aligned}
u(x, t)= & \frac{1}{2}\left(u(x, 0)+u_{t}(x, 0) t+u(a, t)+(x-a) \frac{\partial u(b, t)}{\partial x}\right. \\
& +L_{t t}^{-1} g(x, t)+L_{x x}^{-1} g(x, t)-L_{t t}^{-1} L_{x x} u(x, t)-L_{x x}^{-1} L_{t t} u(x, t) \\
& \left.-L_{t t}^{-1} N u(x, t)-L_{x x}^{-1} N u(x, t)\right)
\end{aligned}
$$

Substituting $u(x, t)=\sum_{n=0}^{\infty} u_{n}(x, t)$, and $N u(x, t)=\sum_{n=0}^{\infty} A_{n}\left(u_{0}, u_{1}, u_{2}, \cdots, u_{n}\right)$ into Equation (4.3), gives

$$
\begin{aligned}
\sum_{n=0}^{\infty} u_{n}(x, t)= & \frac{1}{2}\left(u(x, 0)+u_{t}(x, 0) t+u(a, t)+(x-a) \frac{\partial u(b, t)}{\partial x}\right. \\
& +L_{t t}^{-1} g(x, t)+L_{x x}^{-1} g(x, t)-L_{t t}^{-1} L_{x x} \sum_{n=0}^{\infty} u_{n}(x, t) \\
& \left.-L_{x x}^{-1} L_{t t} \sum_{n=0}^{\infty} u_{n}(x, t)-L_{t t}^{-1} \sum_{n=0}^{\infty} A_{n}(x, t)-L_{x x}^{-1} \sum_{n=0}^{\infty} A_{n}(x, t)\right)
\end{aligned}
$$

so that the recurrence relations are

$$
\begin{gathered}
u_{0}=\frac{1}{2}\left(u(x, 0)+u_{t}(x, 0) t+u(a, t)+(x-a) \frac{\partial u(b, t)}{\partial x}+L_{t t}^{-1} g(x, t)+L_{x x}^{-1} g(x, t)\right) \\
u_{n+1}=\frac{1}{2}\left(-L_{t t}^{-1} L_{x x} u_{n}-L_{x x}^{-1} L_{t t} u_{n}-L_{t t}^{-1} A_{n}-L_{x x}^{-1} A_{n}\right), n \geq 0
\end{gathered}
$$

To give a clear overview of these methods, we have chosen several differential equations. The examples will be approached by the Lesnic's Approach and the modified technique for comparison reasons. We also compare the approximate solution with the exact solution.

\subsection{Numerical Experiments}

\section{Example 4}

Consider the linear homogeneous heat equation

$$
u_{t}-u_{x x}=0,0 \leq x \leq 1, t>0 \text {, }
$$

with specified conditions $u(x, 0)=\mathrm{e}^{x}, u(0, t)=\mathrm{e}^{t}, u_{x}(1, t)=\mathrm{e}^{1+t}$.

Rewriting the heat equation in the operator form as $L_{t} u=L_{x x} u$. Applying the inverse operator $L_{x x}^{-1}$ defined by $L_{x x}^{-1}=\int_{0}^{x} \mathrm{~d} x^{\prime} \int_{1}^{x^{\prime}} \mathrm{d} x^{\prime \prime}$, we get the recursive relations

$$
\begin{gathered}
u_{0}=u(0, t)+x \frac{\partial u(1, t)}{\partial x} \\
u_{n+1}=L_{x x}^{-1} L_{t} u_{n}, n \geq 0
\end{gathered}
$$

So that

$$
\begin{gathered}
u_{0}=\mathrm{e}^{t}+x \mathrm{e}^{1+t}, \\
u_{1}=\frac{1}{6} \mathrm{e}^{1+t} x^{3}+\frac{1}{2} \mathrm{e}^{t} x^{2}-\mathrm{e}^{t} x-\frac{1}{2} \mathrm{e}^{1+t} x,
\end{gathered}
$$




$$
u_{2}=\frac{1}{120} \mathrm{e}^{1+t} x^{5}+\frac{1}{24} \mathrm{e}^{t} x^{4}+\frac{1}{3}\left(\frac{-1}{2} \mathrm{e}^{t}-\frac{1}{4} \mathrm{e}^{1+t}\right) x^{3}+\frac{5}{24} x \mathrm{e}^{1+t}+\frac{1}{3} \mathrm{e}^{t} x,
$$

Continuing in a similar way $u_{2}, u_{3}, \cdots, u_{n}$ are obtained for some $n$, then we get the approximate solution $u_{a p}=\sum_{n=0} u_{n}(x, t)$ which converged to the exact solution $u_{e x}=\mathrm{e}^{x+t}$.

Table 3 shows the comparison between the absolute error of exact and approximate solutions for various values of $t$. Figure 5 and Figure 6 show the results for the exact solution and the approximate solution by application of Lesnic's approach for $t=0.5, t=1.0$.

\section{Example 5}

Consider the linear homogeneous heat equation

$$
u_{t}=u_{x x}+u, t>0
$$

with specified conditions $u(0, t)=\mathrm{e}^{\left(1-\pi^{2}\right) t}, u_{x}(0, t)=0$.

Rewriting the heat equation in the operator form a $L_{t} u=L_{x x} u+u$.

Applying the inverse operator $L_{x x}^{-1}$ defined by $L_{x x}^{-1}=\int_{0}^{x} \mathrm{~d} x^{\prime} \int_{1}^{x^{\prime}} \mathrm{d} x^{\prime \prime}$, we get the recursive relations

$$
\begin{gathered}
u_{0}=u(0, t)+x \frac{\partial u(0, t)}{\partial x}, \\
u_{n+1}=L_{x x}^{-1} L_{t} u_{n}-L_{x x}^{-1} u_{n}, n \geq 0 .
\end{gathered}
$$

So that

$$
\begin{gathered}
u_{0}=\mathrm{e}^{\left(1-\pi^{2}\right) t}, \\
u_{1}=\frac{1}{2}\left(\left(1-\pi^{2}\right) \mathrm{e}^{\left(1-\pi^{2}\right) t}-\mathrm{e}^{\left(1-\pi^{2}\right) t}\right) x^{2}, \\
u_{2}=\frac{1}{4}\left(\frac{1}{6}\left(1-\pi^{2}\right)^{2} \mathrm{e}^{\left(1-\pi^{2}\right) t}-\frac{1}{3}\left(1-\pi^{2}\right) \mathrm{e}^{\left(1-\pi^{2}\right) t}+\frac{1}{6} \mathrm{e}^{\left(1-\pi^{2}\right) t}\right) x^{4},
\end{gathered}
$$

Continuing in a similar way $u_{3}, u_{4}, \cdots, u_{n}$ are obtained for some $n$, then we get the approximate solution $u_{a p}=\sum_{n=0}^{\infty} u_{n}(x, t)$ which converged to the exact solution $u_{e x}=\mathrm{e}^{\left(1-\pi^{2}\right) t} \cos (\pi x)$.

Table 4 shows the comparison between the absolute error of exact and approximate solutions for various values of $t$. Figure 7 and Figure 8 show the results for the exact solution and the approximate solution by application of Lesni's approach for $t=0.5, t=1.0$.

\section{Example 6}

Consider the nonlinear inhomogeneous wave equation

$$
u_{t t}-u_{x x}+u^{2}=2 x^{2}-2 t^{2}+x^{4} t^{4}, t>0
$$

with specified initial condition $u(x, 0)=0, u_{t}(x, 0)=0$ and the boundary conditions $u(0, t)=0, u_{x}(0, t)=0$.

Rewriting the wave equation in the operator form as

$$
L_{t t} u-L_{x x} u+u^{2}=2 x^{2}-2 t^{2}+x^{4} t^{4} .
$$


Table 3. Absolute errors of Lesnic's approach at $t=0.5, t=1.0$.

\begin{tabular}{ccccccc}
\hline$x$ & \multicolumn{3}{c}{$t=0.5$} & & & \multicolumn{1}{c}{$t=1.0$} \\
\cline { 2 - 7 } & $\varphi_{3}$ & $\varphi_{5}$ & $\varphi_{10}$ & $\varphi_{3}$ & $\varphi_{5}$ & $\varphi_{10}$ \\
\hline 0.0 & $0.00000000 \mathrm{e}+00$ & $0.00000000 \mathrm{e}+00$ & $0.00000000 \mathrm{e}+00$ & $0.00000000 \mathrm{e}+00$ & $0.00000000 \mathrm{e}+00$ & $0.00000000 \mathrm{e}+00$ \\
0.1 & $4.24902315 \mathrm{e}-02$ & $6.97705740 \mathrm{e}-03$ & $7.62907729 \mathrm{e}-05$ & $7.00545484 \mathrm{e}-02$ & $1.15032229 \mathrm{e}-02$ & $1.25782220 \mathrm{e}-04$ \\
0.2 & $8.39308011 \mathrm{e}-02$ & $1.37823116 \mathrm{e}-02$ & $1.50703014 \mathrm{e}-04$ & $1.38378497 \mathrm{e}-01$ & $2.27231903 \mathrm{e}-02$ & $2.48467264 \mathrm{e}-04$ \\
0.3 & $1.23299225 \mathrm{e}-01$ & $2.02481910 \mathrm{e}-02$ & $2.21404446 \mathrm{e}-04$ & $2.03286055 \mathrm{e}-01$ & $3.33836232 \mathrm{e}-02$ & $3.65034220 \mathrm{e}-04$ \\
0.4 & $1.59625814 \mathrm{e}-01$ & $2.62154825 \mathrm{e}-02$ & $2.86654167 \mathrm{e}-04$ & $2.63178475 \mathrm{e}-01$ & $4.32220236 \mathrm{e}-02$ & $4.72612822 \mathrm{e}-04$ \\
0.5 & $1.92017276 \mathrm{e}-01$ & $3.15372522 \mathrm{e}-02$ & $3.44845510 \mathrm{e}-04$ & $3.16582967 \mathrm{e}-01$ & $5.19961385 \mathrm{e}-02$ & $5.68554127 \mathrm{e}-04$ \\
0.6 & $2.19678089 \mathrm{e}-01$ & $3.60824631 \mathrm{e}-02$ & $3.94545612 \mathrm{e}-04$ & $3.62187938 \mathrm{e}-01$ & $5.94899244 \mathrm{e}-02$ & $6.50495743 \mathrm{e}-04$ \\
0.7 & $2.41929405 \mathrm{e}-01$ & $3.97392012 \mathrm{e}-02$ & $4.34530692 \mathrm{e}-04$ & $3.98874156 \mathrm{e}-01$ & $6.55188663 \mathrm{e}-02$ & $7.16419995 \mathrm{e}-04$ \\
0.8 & $2.58225232 \mathrm{e}-01$ & $4.24174302 \mathrm{e}-02$ & $4.63816184 \mathrm{e}-04$ & $4.25741433 \mathrm{e}-01$ & $6.99345194 \mathrm{e}-02$ & $7.64703609 \mathrm{e}-04$ \\
0.9 & $2.68165604 \mathrm{e}-01$ & $4.40512072 \mathrm{e}-02$ & $4.81680983 \mathrm{e}-04$ & $4.42130336 \mathrm{e}-01$ & $7.26281622 \mathrm{e}-02$ & $7.94157682 \mathrm{e}-04$ \\
1.0 & $2.71506419 \mathrm{e}-01$ & $4.46003060 \mathrm{e}-02$ & $4.87685197 \mathrm{e}-04$ & $4.47638409 \mathrm{e}-01$ & $7.35334732 \mathrm{e}-02$ & $8.04056958 \mathrm{e}-04$ \\
\hline
\end{tabular}

Table 4. Absolute errors of Lesnic's approach at $t=0.5, t=1.0$.

\begin{tabular}{|c|c|c|c|c|c|c|}
\hline \multirow{2}{*}{$x$} & \multicolumn{3}{|c|}{$t=0.5$} & \multicolumn{3}{|c|}{$t=1.0$} \\
\hline & $\varphi_{3}$ & $\varphi_{5}$ & $\varphi_{10}$ & $\varphi_{3}$ & $\varphi_{5}$ & $\varphi_{10}$ \\
\hline 0.0 & $0.00000000 \mathrm{e}+00$ & $0.00000000 \mathrm{e}+00$ & $0.00000000 \mathrm{e}+00$ & $0.00000000 \mathrm{e}+00$ & $0.00000000 \mathrm{e}+00$ & $0.00000000 \mathrm{e}+00$ \\
\hline 0.1 & $1.58048860 \mathrm{e}-08$ & $3.05774246 \mathrm{e}-14$ & $4.00000000 \mathrm{e}-31$ & $1.87405030 \mathrm{e}-10$ & $3.62569093 e-16$ & $4.00000000 \mathrm{e}-33$ \\
\hline 0.2 & $1.00618440 \mathrm{e}-06$ & $3.12411864 \mathrm{e}-11$ & $4.47807730 \mathrm{e}-25$ & $1.19307420 \mathrm{e}-08$ & $3.70439590 \mathrm{e}-13$ & $5.30984100 \mathrm{e}-27$ \\
\hline 0.3 & $1.13607976 \mathrm{e}-05$ & $1.79481652 \mathrm{e}-09$ & $1.48748759 \mathrm{e}-21$ & $1.34709647 \mathrm{e}-07$ & $2.12818773 \mathrm{e}-11$ & $1.76377518 \mathrm{e}-23$ \\
\hline 0.4 & $6.30539648 \mathrm{e}-05$ & $3.17061819 \mathrm{e}-08$ & $4.68360013 e-19$ & $7.47656780 \mathrm{e}-07$ & $3.75953232 \mathrm{e}-10$ & $5.55353720 \mathrm{e}-21$ \\
\hline 0.5 & $2.36780141 \mathrm{e}-04$ & $2.93320054 \mathrm{e}-07$ & $4.05459549 \mathrm{e}-17$ & $2.80759946 \mathrm{e}-06$ & $3.47801646 \mathrm{e}-09$ & $4.80770055 \mathrm{e}-19$ \\
\hline 0.6 & $6.93626458 \mathrm{e}-04$ & $1.80143646 \mathrm{e}-06$ & $1.55080207 \mathrm{e}-15$ & $8.22461404 \mathrm{e}-06$ & $2.13603726 \mathrm{e}-08$ & $1.83884976 \mathrm{e}-17$ \\
\hline 0.7 & $1.71018201 \mathrm{e}-03$ & $8.33538786 \mathrm{e}-06$ & $3.37515258 \mathrm{e}-14$ & $2.02783311 \mathrm{e}-05$ & $9.88361203 e-08$ & $4.00205716 \mathrm{e}-16$ \\
\hline 0.8 & $3.71364715 \mathrm{e}-03$ & $3.13376896 \mathrm{e}-05$ & $4.86129475 \mathrm{e}-13$ & $4.40342408 \mathrm{e}-05$ & $3.71583868 \mathrm{e}-07$ & $5.76423702 \mathrm{e}-15$ \\
\hline 0.9 & $7.31350601 \mathrm{e}-03$ & $1.00509330 \mathrm{e}-04$ & $5.10789012 \mathrm{e}-12$ & $8.67192471 \mathrm{e}-05$ & $1.19178044 \mathrm{e}-06$ & $6.05663528 \mathrm{e}-14$ \\
\hline 1.0 & $1.33266620 \mathrm{e}-02$ & $2.84314481 \mathrm{e}-04$ & $4.18457517 \mathrm{e}-11$ & $1.58019709 \mathrm{e}-04$ & $3.37123368 \mathrm{e}-06$ & $4.96182279 \mathrm{e}-13$ \\
\hline
\end{tabular}
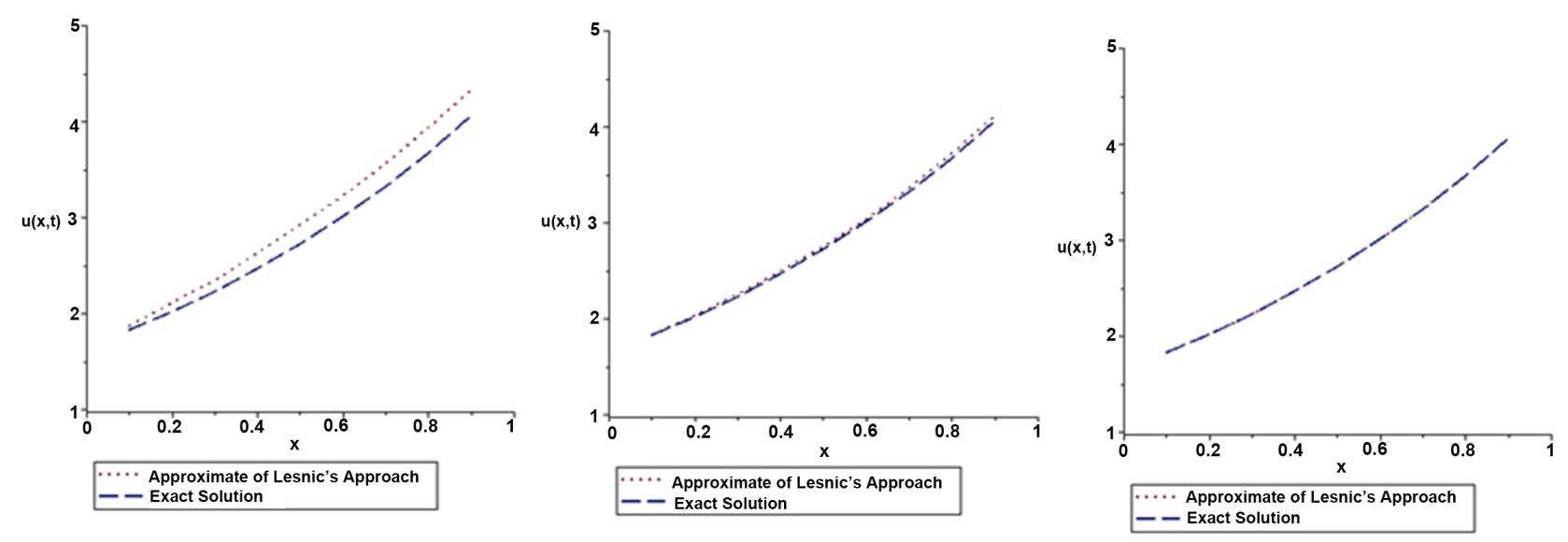

Figure 5. The exact solution and the approximate solution of Lesnic's approach for $\varphi_{3}, \varphi_{5}, \varphi_{10}$ at $t=0.5$. 

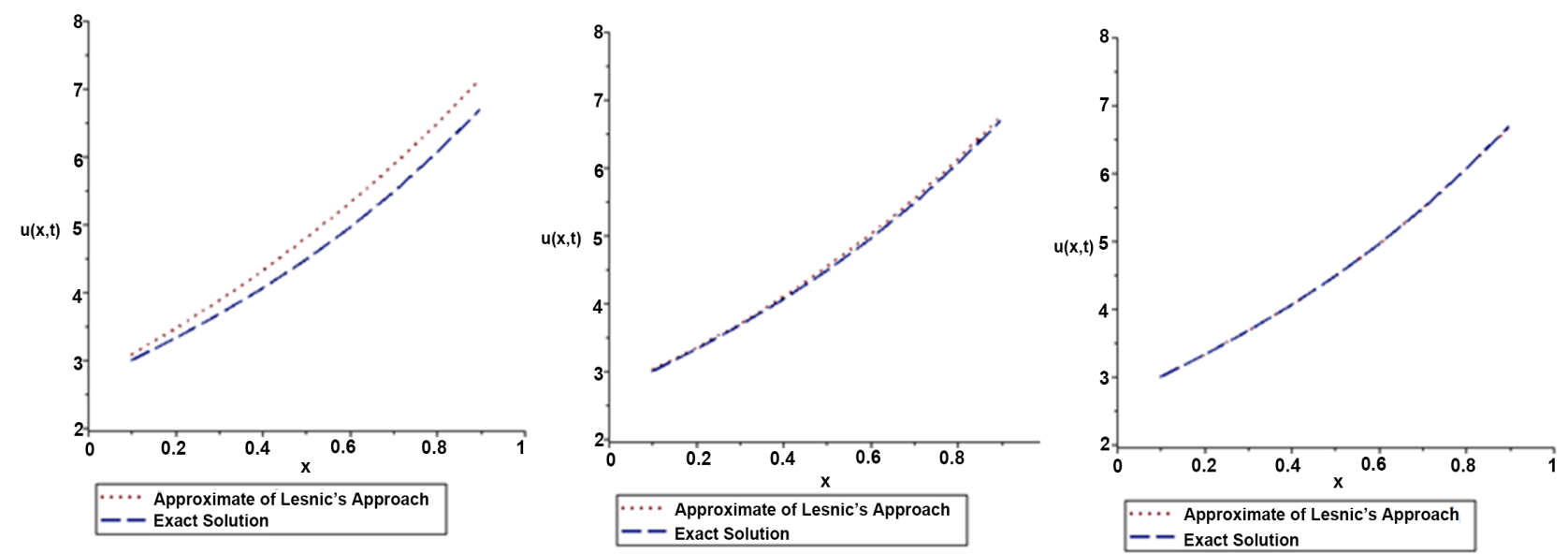

Figure 6. The exact solution and the approximate solution of Lesnic's approach for $\varphi_{3}, \varphi_{5}, \varphi_{10}$ at $t=1.0$.
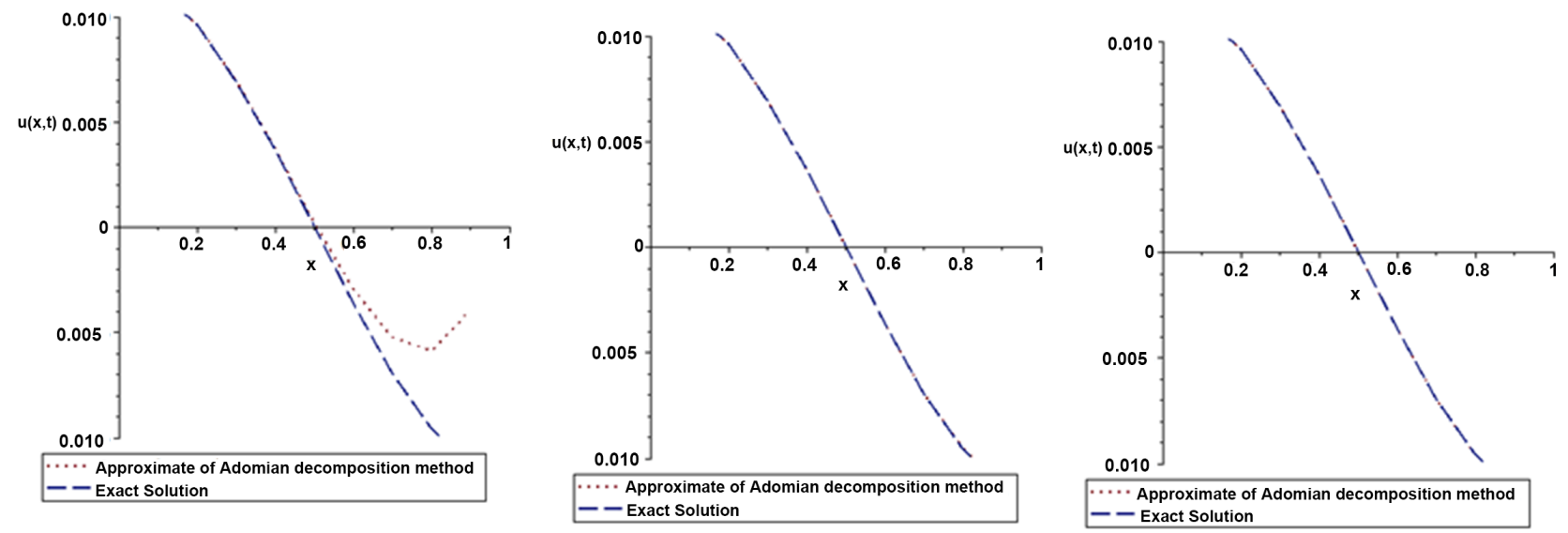

Figure 7. The exact solution and the approximate solution of Lesnic's approach for $\varphi_{3}, \varphi_{5}, \varphi_{10}$ at $t=0.5$.
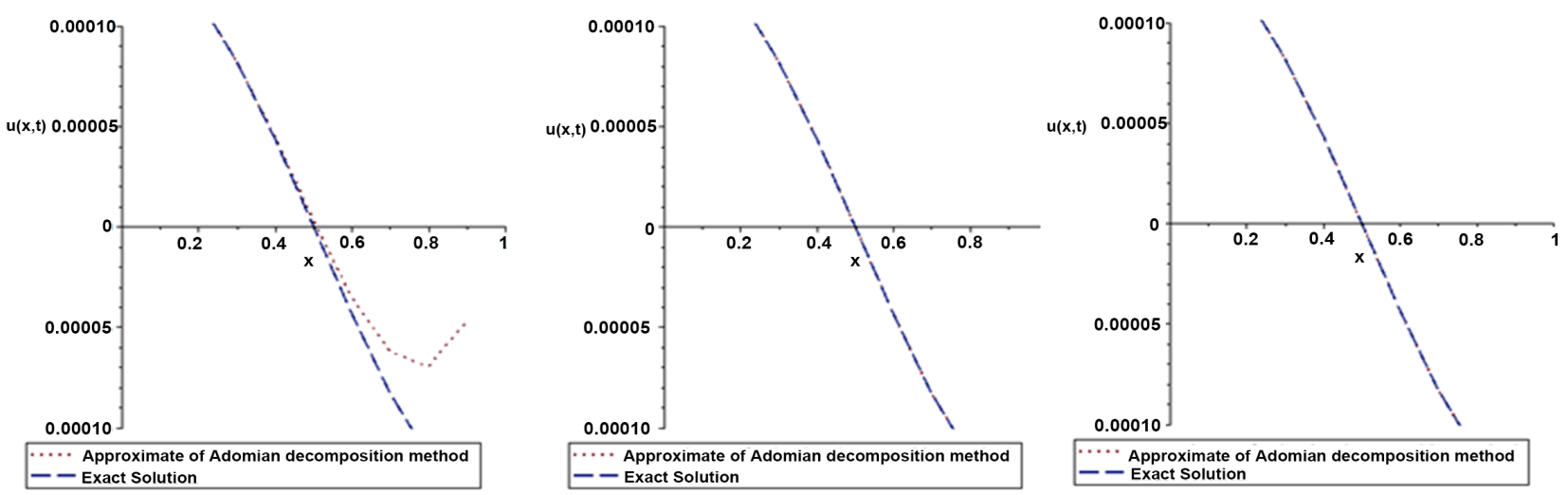

Figure 8. The exact solution and the approximate solution of Lesnic's approach for $\varphi_{3}, \varphi_{5}, \varphi_{10}$ at $t=1.0$.

Firstly, we consider the $t$ partial solution as

$$
L_{t t} u=L_{x x} u-u^{2}+2 x^{2}-2 t^{2}+x^{4} t^{4} .
$$

Applying the inverse operator $L_{t t}^{-1}$ defined by $L_{t t}^{-1}=\int_{0}^{t} \int_{0}^{t} \mathrm{~d} t \mathrm{~d} t$, gives the recursive relations 


$$
\begin{gathered}
u_{0}=u(x, 0)+t u_{t}(x, 0)+L_{t t}^{-1}\left(2 x^{2}-2 t^{2}+x^{4} t^{4}\right), \\
u_{n+1}=L_{t t}^{-1} L_{x x} u_{n}-L_{t t}^{-1} A_{n}, n \geq 0 .
\end{gathered}
$$

So that

$$
\begin{gathered}
u_{0}=\frac{1}{30} x^{4} t^{6}-\frac{1}{6} t^{4}+x^{2} t^{2}, \\
u_{1}=\frac{11}{840} x^{2} t^{8}+\frac{1}{6} t^{4}-\frac{1}{163800} x^{8} t^{14}+\frac{1}{11880} x^{4} t^{12} \\
-\frac{1}{10}\left(\frac{1}{135} x^{6}+\frac{1}{324}\right) t^{10}-\frac{1}{30} x^{4} t^{6},
\end{gathered}
$$

Secondly, we consider the $x$ partial solution

$$
L_{x x} u=L_{t t} u+u^{2}-2 x^{2}+2 t^{2}-x^{4} t^{4} .
$$

Applying the inverse operator $L_{x x}^{-1}$ defined by $L_{x x}^{-1}=\int_{0}^{x} \mathrm{~d} x^{\prime} \int_{0}^{x^{\prime}} \mathrm{d} x^{\prime \prime}$, gives the recursive relations

$$
\begin{gathered}
u_{0}=u(0, t)+x \frac{\partial u(0, t)}{\partial x}+L_{x x}^{-1}\left(-2 x^{2}+2 t^{2}-x^{4} t^{4}\right), \\
u_{n+1}=L_{x x}^{-1} L_{t} u_{n}-L_{x x}^{-1} A_{n}, n \geq 0 .
\end{gathered}
$$

So that

$$
\begin{gathered}
u_{0}=-\frac{1}{30} t^{4} x^{6}-\frac{1}{6} x^{4}+x^{2} t^{2}, \\
u_{1}=-\frac{11}{840} t^{2} x^{8}+\frac{1}{6} x^{4}+\frac{1}{163800} t^{8} x^{14}+\frac{1}{11880} t^{4} x^{12} \\
+\frac{1}{10}\left(\frac{-1}{135} t^{6}+\frac{1}{324}\right) x^{10}+\frac{1}{30} t^{4} x^{6},
\end{gathered}
$$

Next, we average the partial solutions, i.e. add two partial solutions and divide by two, so we obtain

$$
\begin{aligned}
u_{0}= & \frac{1}{2}\left(\frac{1}{30} x^{4} t^{6}-\frac{1}{6} t^{4}+2 x^{2} t^{2}-\frac{1}{30} t^{4} x^{6}-\frac{1}{6} x^{4}\right), \\
u_{1}= & \frac{1}{2}\left(\frac{11}{840} x^{2} t^{8}+\frac{1}{6} t^{4}-\frac{1}{163800} x^{8} t^{14}+\frac{1}{11880} x^{4} t^{12}\right. \\
& -\frac{1}{10}\left(\frac{1}{135} x^{6}+\frac{1}{324}\right) t^{10}-\frac{1}{30} x^{4} t^{6}-\frac{11}{840} t^{2} x^{8} \\
& +\frac{1}{6} x^{4}+\frac{1}{163800} t^{8} x^{14}+\frac{1}{11880} t^{4} x^{12} \\
& \left.+\frac{1}{10}\left(\frac{-1}{135} t^{6}+\frac{1}{324}\right) x^{10}+\frac{1}{30} t^{4} x^{6}\right),
\end{aligned}
$$

Continuing in a similar way $u_{2}, u_{3}, \cdots, u_{n}$ are obtained for some $n$, then we get the approximate solution $u_{a p}=\sum_{n=0}^{\infty} u_{n}(x, t)$ which converged to the exact solution $u_{e x}=x^{2} t^{2}$.

Table 5 shows the comparison between the absolute error of exact and approximate solutions for various values of $t$. Figure 9 and Figure 10 show the 
Table 5. Absolute errors of Lesnic's approach at $t=0.5, t=1.0$.

\begin{tabular}{ccccccc}
\hline \multirow{2}{*}{$x$} & & $t=0.5$ & & & \multicolumn{1}{c}{$t=1.0$} & \\
\cline { 3 - 6 } & $\varphi_{3}$ & $\varphi_{5}$ & $\varphi_{10}$ & $\varphi_{3}$ & $\varphi_{5}$ & $\varphi_{10}$ \\
\cline { 2 - 6 } 0.0 & $2.92793246 \mathrm{e}-07$ & $1.01879027 \mathrm{e}-11$ & $6.85095911 \mathrm{e}-25$ & $2.99609298 \mathrm{e}-04$ & $6.66358850 \mathrm{e}-07$ & $1.17411886 \mathrm{e}-14$ \\
0.1 & $2.92797760 \mathrm{e}-07$ & $1.01884440 \mathrm{e}-11$ & $5.49993680 \mathrm{e}-25$ & $2.99751384 \mathrm{e}-04$ & $6.67335460 \mathrm{e}-07$ & $6.46368619 \mathrm{e}-15$ \\
0.2 & $2.92714604 \mathrm{e}-07$ & $1.01838277 \mathrm{e}-11$ & $4.25441720 \mathrm{e}-24$ & $2.99906957 \mathrm{e}-04$ & $6.68628865 \mathrm{e}-07$ & $9.47122029 \mathrm{e}-15$ \\
0.3 & $2.90660076 \mathrm{e}-07$ & $1.01582155 \mathrm{e}-11$ & $1.04069553 \mathrm{e}-23$ & $2.99261398 \mathrm{e}-04$ & $6.65318536 \mathrm{e}-07$ & $3.60495597 \mathrm{e}-14$ \\
0.4 & $2.59880611 \mathrm{e}-07$ & $1.03640472 \mathrm{e}-11$ & $1.88856065 \mathrm{e}-23$ & $2.96428943 \mathrm{e}-04$ & $6.49193205 \mathrm{e}-07$ & $7.21721640 \mathrm{e}-14$ \\
0.5 & $6.38490216 \mathrm{e}-09$ & $2.03770775 \mathrm{e}-11$ & $1.39263400 \mathrm{e}-24$ & $2.89265750 \mathrm{e}-04$ & $6.08842328 \mathrm{e}-07$ & $1.14059558 \mathrm{e}-13$ \\
0.6 & $1.55441030 \mathrm{e}-06$ & $2.05585573 \mathrm{e}-10$ & $1.04956960 \mathrm{e}-20$ & $2.73991554 \mathrm{e}-04$ & $5.30208113 \mathrm{e}-07$ & $1.53190969 \mathrm{e}-13$ \\
0.7 & $8.35705522 \mathrm{e}-06$ & $2.34523011 \mathrm{e}-09$ & $1.45619577 \mathrm{e}-18$ & $2.42057261 \mathrm{e}-04$ & $4.00247226 \mathrm{e}-07$ & $1.74249490 \mathrm{e}-13$ \\
0.8 & $3.27155910 \mathrm{e}-05$ & $2.00278856 \mathrm{e}-08$ & $1.03741414 \mathrm{e}-16$ & $1.70952966 \mathrm{e}-04$ & $2.32477815 \mathrm{e}-07$ & $1.53300820 \mathrm{e}-13$ \\
0.9 & $1.07359772 \mathrm{e}-04$ & $1.33275317 \mathrm{e}-07$ & $4.44838968 \mathrm{e}-15$ & $8.65289576 \mathrm{e}-07$ & $2.20560700 \mathrm{e}-07$ & $2.08420940 \mathrm{e}-14$ \\
1.0 & $3.09754605 \mathrm{e}-04$ & $7.26354463 \mathrm{e}-07$ & $1.27588322 \mathrm{e}-13$ & $4.18259832 \mathrm{e}-04$ & $1.50572369 \mathrm{e}-06$ & $1.53639602 \mathrm{e}-12$ \\
\hline
\end{tabular}
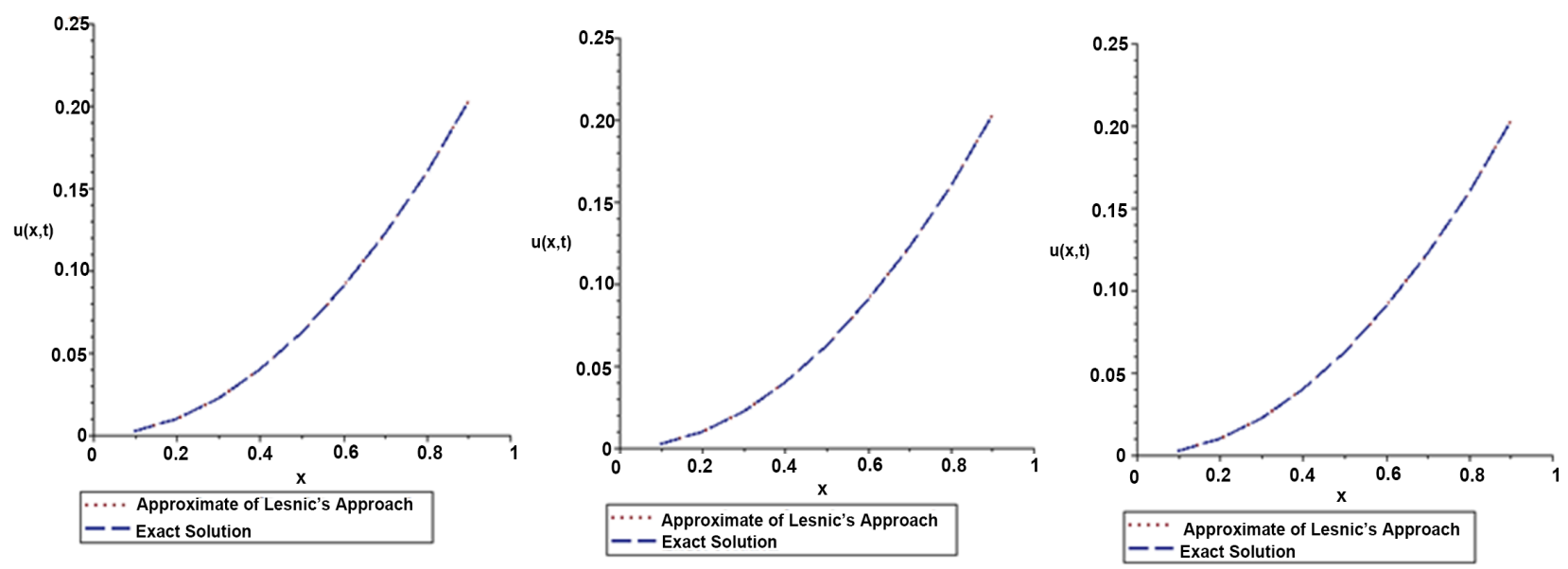

Figure 9. The exact solution and the approximate solution of Lesnic's approach for $\varphi_{3}, \varphi_{5}, \varphi_{10}$ at $t=0.5$.
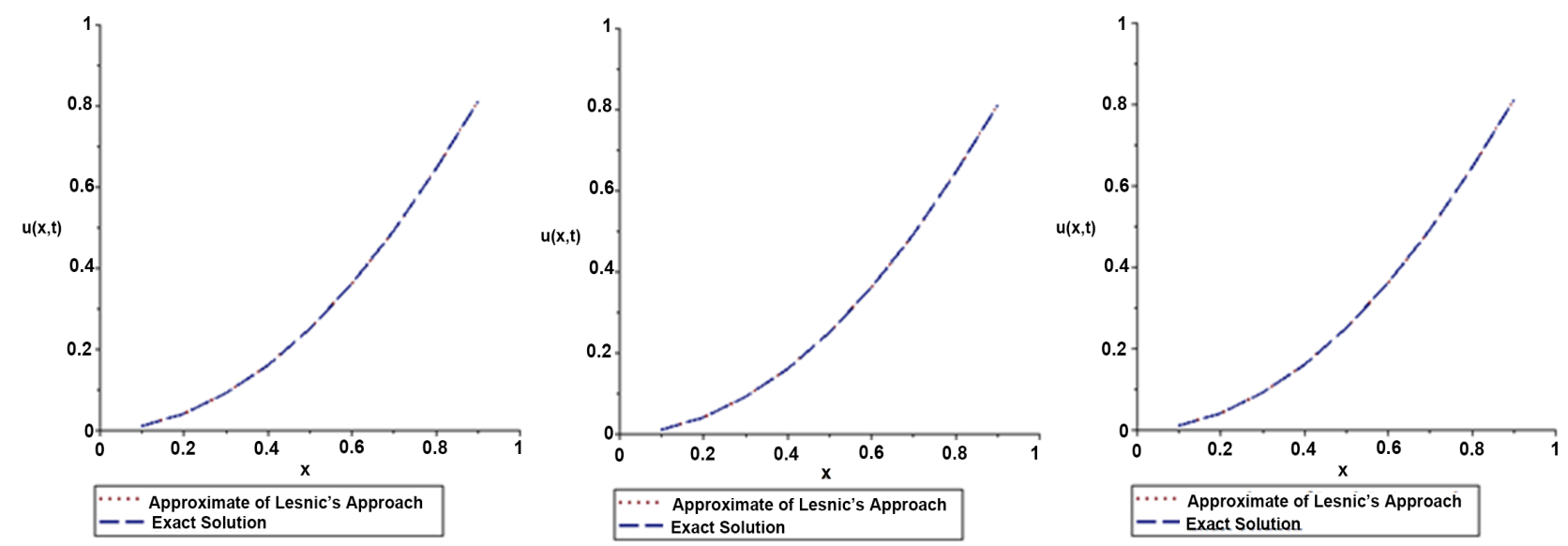

Figure 10. The exact solution and the approximate solution of Lesnic's approach for $\varphi_{3}, \varphi_{5}, \varphi_{10}$ at $t=1.0$. 
results for the exact solution and the approximate solution by application of Lesnic's approach for $t=0.5, t=1.0$.

\section{Example 7}

Consider the nonlinear inhomogeneous heat equation

$$
u_{t}=u_{x x}+u^{2}-u-t^{2} \mathrm{e}^{2 x}+\mathrm{e}^{x}, t>0,
$$

with specified conditions $u(x, 0)=0, u(0, t)=t, u_{x}(0, t)=t$. Rewriting the heat equation in the operator form as

$$
L_{t} u=L_{x x} u+u^{2}-u-t^{2} \mathrm{e}^{2 x}+\mathrm{e}^{x} .
$$

Applying the inverse operator $L_{x x}^{-1}$ defined by $L_{x x}^{-1}=\int_{0}^{x} \mathrm{~d} x^{\prime} \int_{0}^{x^{\prime}} \mathrm{d} x^{\prime \prime}$, we get the recursive relations

$$
\begin{gathered}
u_{0}=u(0, t)+x \frac{\partial u(0, t)}{\partial x}+L_{x x}^{-1}\left(t^{2} \mathrm{e}^{2 x}-\mathrm{e}^{x}\right), \\
u_{n+1}=L_{x x}^{-1} L_{t} u_{n}+L_{x x}^{-1} u_{n}-L_{x x}^{-1} A_{n}, n \geq 0 .
\end{gathered}
$$

So that

$$
u_{0}=t+x t+1-\frac{1}{4} t^{2}-\frac{1}{2} t^{2} x+x+\frac{1}{4} t^{2} \mathrm{e}^{2 x}-\mathrm{e}^{x},
$$

We set $u_{0}=f$ then we divide it into two parts, so that

$$
\begin{gathered}
u_{0}=f_{1}=t+x t, \\
u_{1}=f_{2}+L_{x x}^{-1} L_{t} u_{0}+L_{x x}^{-1} u_{0}-L_{x x}^{-1} A_{0} \\
=\left(1-\frac{1}{4} t^{2}-\frac{1}{2} t^{2} x+x+\frac{1}{4} t^{2} \mathrm{e}^{2 x}-\mathrm{e}^{x}\right)+\frac{1}{6} x^{3}+\frac{1}{2} x^{2} \\
-\frac{1}{12} t^{2} x^{4}-\frac{1}{3} t^{2} x^{3}-\frac{1}{2} t^{2} x^{2}+\frac{1}{6} t x^{3}+\frac{1}{2} t x^{2},
\end{gathered}
$$

Continuing in a similar way $u_{2}, u_{3}, \cdots, u_{n}$ are obtained for some $n$, then we get the approximate solution $u_{a p}=\sum_{n=0}^{\infty} u_{n}(x, t)$ which converged to the exact solution $u_{e x}=t \mathrm{e}^{x}$.

Table 6 shows the comparison between the absolute error of exact and approximate solutions Lesnic's approach and modified of Lesnic's approach for various values of $t$. Figure 11 and Figure 12 show the results for the exact solution and the approximate solution by application of Lesnic's approach and modified of Lesnic's Approach for $t=0.5$.

\section{Conclusion}

The main goal of this paper is the development of an efficient and accurate method to solve linear and nonlinear initial-boundary value problems with mixed boundary conditions. The method developed provides the solution in the form of rapidly convergent series relative to the Adomian decomposition method. It also introduces a slight change in the formulation of Adomian recursive relation. The method presented is applied to several examples of boundary-value problems and initial boundary-value problems with mixed type boundary conditions. 
Table 6. Absolute errors of Lesnic's approach and modified of Lesnic's Approach at $t=0.5$.

\begin{tabular}{|c|c|c|c|c|c|c|}
\hline \multirow{2}{*}{$x$} & \multicolumn{3}{|c|}{ Lesnic's Approach } & \multicolumn{3}{|c|}{ modified of Lesnic's Approach } \\
\hline & $\varphi_{3}$ & $\varphi_{5}$ & $\varphi_{10}$ & $\varphi_{3}$ & $\varphi_{5}$ & $\varphi_{10}$ \\
\hline 0.1 & $2.00447357 \mathrm{e}-08$ & $3.57237376 \mathrm{e}-14$ & $1.37823812 \mathrm{e}-10$ & $3.81571653 e-09$ & $1.06888605 \mathrm{e}-16$ & $1.24058539 \mathrm{e}-17$ \\
\hline 0.2 & $1.33381463 \mathrm{e}-06$ & $3.85833086 \mathrm{e}-11$ & $8.00849170 \mathrm{e}-11$ & $2.67012987 \mathrm{e}-07$ & $3.14421657 \mathrm{e}-13$ & $1.83039464 \mathrm{e}-16$ \\
\hline 0.3 & $1.57920700 \mathrm{e}-05$ & $2.34517027 \mathrm{e}-09$ & $1.19984480 \mathrm{e}-11$ & $3.31116702 \mathrm{e}-06$ & $4.40980463 \mathrm{e}-11$ & $5.19918722 \mathrm{e}-17$ \\
\hline 0.4 & $9.22165384 \mathrm{e}-05$ & $4.38853494 \mathrm{e}-08$ & $7.93648411 \mathrm{e}-11$ & $2.01789433 \mathrm{e}-05$ & $1.27255431 \mathrm{e}-09$ & $2.58912426 \mathrm{e}-16$ \\
\hline 0.5 & $3.65597018 \mathrm{e}-04$ & $4.30762657 \mathrm{e}-07$ & $1.49935927 \mathrm{e}-10$ & $8.32226565 \mathrm{e}-05$ & $1.66889704 \mathrm{e}-08$ & $7.73424325 \mathrm{e}-16$ \\
\hline 0.6 & $1.13467005 \mathrm{e}-03$ & $2.81244230 \mathrm{e}-06$ & $1.95254487 \mathrm{e}-10$ & $2.67910691 \mathrm{e}-04$ & $1.35122395 \mathrm{e}-07$ & $1.65874377 \mathrm{e}-14$ \\
\hline 0.8 & $6.89226862 \mathrm{e}-03$ & $5.56852385 \mathrm{e}-05$ & $2.49626415 \mathrm{e}-10$ & $1.73726451 \mathrm{e}-03$ & $3.63635665 \mathrm{e}-06$ & $7.09170229 \mathrm{e}-12$ \\
\hline 0.9 & $1.45351895 \mathrm{e}-02$ & $1.91314569 \mathrm{e}-04$ & $1.32152517 \mathrm{e}-09$ & $3.77230615 \mathrm{e}-03$ & $1.40126738 \mathrm{e}-05$ & $8.56616462 \mathrm{e}-11$ \\
\hline 1.0 & $2.84628000 \mathrm{e}-02$ & $5.81448247 \mathrm{e}-04$ & $1.18704774 \mathrm{e}-08$ & $7.59013963 \mathrm{e}-03$ & $4.69348011 \mathrm{e}-05$ & $8.00241318 \mathrm{e}-10$ \\
\hline
\end{tabular}
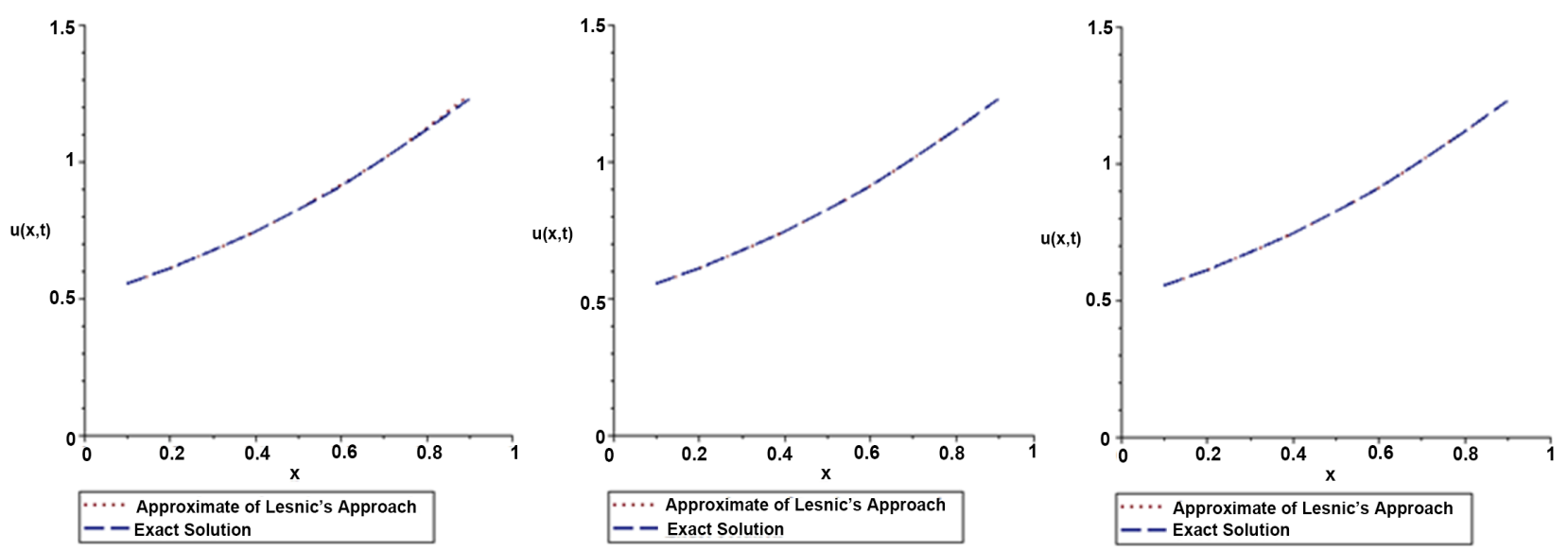

Figure 11. The exact solution and the approximate solution of Lesnic's approach for $\varphi_{3}, \varphi_{5}, \varphi_{10}$ at $t=0.5$.
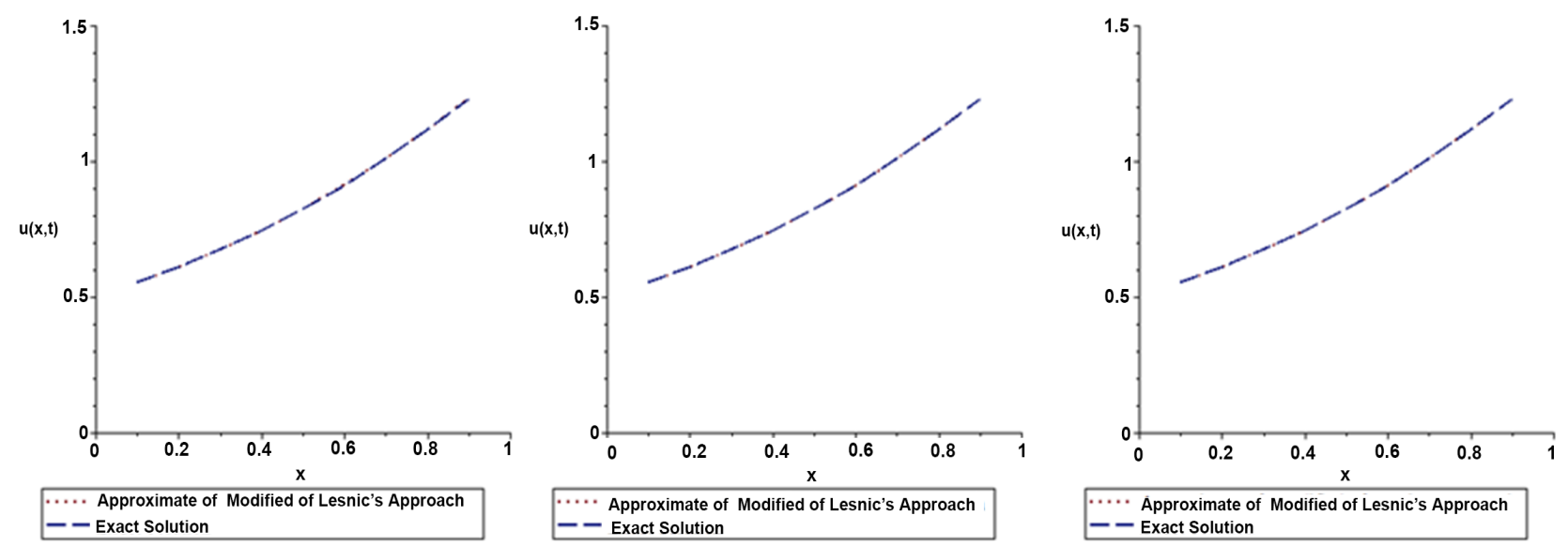

Figure 12. The exact solution and the approximate solution of Modified of Lesnic's approach for $\varphi_{3}, \varphi_{5}, \varphi_{10}$ at $t=0.5$. 
The accuracy and computational efficiency of the proposed method are verified by numerical examples. Thus, the method is highly recommended for varieties of mixed boundary value problems.

\section{References}

[1] Carslaw, H.S. and Jaeger, J.C. (1948) Conduction of Heat in Solids. Clarendon Press, Oxford.

[2] Carrier, G.F., Krook, M. and Pearson, C.E. (1966) Functions of a Complex Variable. McGraw-Hill, New York.

[3] Courant, R. and Hilbert, D. (1953) Methods of Mathematical Physics. Vol. 1, Interscience Publishers Inc., New York.

[4] Sneddon, I.N. (1966) Mixed Boundary Value Problems in Potential Theory. Wiley, New York.

[5] Tranter, C.J. (1951) Integral Transforms in Mathematical Physics. Wiley, New York.

[6] Fabrikant, V.I. (1991) Mixed Boundary Value Problems of Potential Theory and their Applications in Engineering. Kluwer, Boston.

[7] Sherwood, J.D. and Stone, H.A. (2001) Leakage through Filtercake into a Fluid Sampling Probe. Physics of Fluids, 13, 1151-1159. https://doi.org/10.1063/1.1360712

[8] Warrick, A.W., Broadbridge, P. and Lomen, D.O. (1992) Approximations for Diffusion from a Disc Source. Applied Mathematical Modelling, 16, 155-161. https://doi.org/10.1016/0307-904X(92)90067-D

[9] He, J.H. (2008) An Elementary Introduction to the Homotopy Perturbation Method. Computers \& Mathematics with Applications, 57, 410-412. https://doi.org/10.1016/j.camwa.2008.06.003

[10] Wazwaz, A.M. (2006) The Modified Decomposition Method for Analytic Treatment of Differential Equations. Applied Mathematics and Computation, 173, 165-176. https://doi.org/10.1016/j.amc.2005.02.048

[11] Duan, J.S. and Rach, R. (2011) A New Modification of the Adomian Decomposition Method for Solving Boundary Value Problems for Higher Order Nonlinear Differential Equations. Applied Mathematics and Computation, 218, 4090-4118. https://doi.org/10.1016/j.amc.2011.09.037

[12] Adomian, G. and Rach, R. (1983) Inversion of Nonlinear Stochastic Operators. Journal of Mathematical Analysis and Applications, 91, 39-46. https://doi.org/10.1016/0022-247X(83)90090-2

[13] Adomian, G. (1986) Nonlinear Stochastic Operator Equations. Academic, Orlando.

[14] Adomian, G. (1989) Nonlinear Stochastic Systems Theory and Applications to Physics. Kluwer Academic, Dordrecht. https://doi.org/10.1007/978-94-009-2569-4

[15] Adomian, G., Rach, R. and Meyers, R. (1991) An Efficient Methodology for the Physical Sciences. Kybernetes, 20, 24-34. https://doi.org/10.1108/eb005909

[16] Adomian, G. and Rach, R. (1993) Analytic Solution of Nonlinear Boundary Value Problems in Several Dimensions by Decomposition. Journal of Mathematical Analysis and Applications, 174, 118-137. https://doi.org/10.1006/jmaa.1993.1105

[17] Adomian, G. and Rach, R. (1993) A New Algorithm for Matching Boundary Conditions in Decomposition Solutions. Applied Mathematics and Computation, 57, 61-68. https://doi.org/10.1016/0096-3003(93)90012-4

[18] Adomian, G. and Rach, R. (1994) Modified Decomposition Solution of Linear and 
Nonlinear Boundary-Value Problems. Nonlinear Analysis, 23, 615-619. https://doi.org/10.1016/0362-546X(94)90240-2

[19] Adomian, G. (1994) Solving Frontier Problems of Physics: The Decomposition Method. Kluwer Academic, Dordrecht. https://doi.org/10.1007/978-94-015-8289-6

[20] Wazwaz, A.M. (2000) Approximate Solutions to Boundary Value Problems of Higher Order by the Modified Decomposition Method. Computers \& Mathematics with Applications, 40, 679-691. https://doi.org/10.1016/S0898-1221(00)00187-5

[21] Wazwaz, A.M. (2000) The Modified Adomian Decomposition Method for Solving Linear and Nonlinear Boundary Value Problems of 10th-Order and 12th-Order. International Journal of Nonlinear Sciences and Numerical Simulation, 1, 17-24. https://doi.org/10.1515/IJNSNS.2000.1.1.17

[22] Wazwaz, A.M. (2001) A Reliable Algorithm for Obtaining Positive Solutions for Nonlinear Boundary Value Problems. Computers \& Mathematics with Applications, 41, 1237-1244. https://doi.org/10.1016/S0898-1221(01)00094-3

[23] Wazwaz, A.M. (2001) The Numerical Solution of Fifth-Order Boundary Value Problems by the Decomposition Method. Journal of Computational and Applied Mathematics, 136, 259-270. https://doi.org/10.1016/S0377-0427(00)00618-X

[24] Wazwaz, A.M. (2001) The Numerical Solution of Sixth-Order Boundary Value Problems by the Modified Decomposition Method. Applied Mathematics and Computation, 118, 311-325. https://doi.org/10.1016/S0096-3003(99)00224-6

[25] Wazwaz, A.M. (2001) A Reliable Algorithm for Solving Boundary Value Problems for Higher-Order Integro-Differential Equations. Applied Mathematics and Computation, 118, 327-342. https://doi.org/10.1016/S0096-3003(99)00225-8

[26] Wazwaz, A.M. (2002) The Numerical Solution of Special Fourth-Order Boundary Value Problems by the Modified Decomposition Method. International Journal of Computer Mathematics, 79, 345-356. https://doi.org/10.1080/00207160211928

[27] Jang, B. (2008) Two-Point Boundary Value Problems by the Extended Adomian Decomposition Method. Journal of Computational and Applied Mathematics, 219, 253-262. https://doi.org/10.1016/j.cam.2007.07.036

[28] Ebaid, A.E. (2010) Exact Solutions for a Class of Nonlinear Singular Two-Point Boundary Value Problems: The Decomposition Method. Zeitschrift für Naturforschung, 65a, 1-6. https://doi.org/10.1515/zna-2010-0301

[29] Adomian, G. (1986) A New Approach to the Heat Equation-An Application of the Decomposition Method. Journal of Mathematical Analysis and Applications, 113, 202-209. https://doi.org/10.1016/0022-247X(86)90344-6

[30] Lesnic, D. and Elliott, L. (1999) The Decomposition Approach to Inverse Heat Conduction. Journal of Mathematical Analysis and Applications, 232, 82-98. https://doi.org/10.1006/jmaa.1998.6243

[31] Wazwaz, A.M. (1999) A Reliable Modification of Adomian's Decomposition Method. Applied Mathematics and Computation, 102, 77-86.

https://doi.org/10.1016/S0096-3003(98)10024-3

[32] Lesnic, D. (2001) A Computational Algebraic Investigation of the Decomposition Method for Time-Dependent Problems. Applied Mathematics and Computation, 119, 197-206. https://doi.org/10.1016/S0096-3003(99)00257-X 